\title{
Finitary random interlacements and the Gaboriau-Lyons problem
}

\author{
Lewis Bowen* \\ University of Texas at Austin \\ March 7, 2019
}

\begin{abstract}
The von Neumann-Day problem asks whether every non-amenable group contains a non-abelian free group. It was answered in the negative by Ol'shanskii in the 1980s. The measurable version (formulated by Gaboriau-Lyons) asks whether every non-amenable measured equivalence relation contains a non-amenable treeable subequivalence relation. This paper obtains a positive answer in the case of arbitrary Bernoulli shifts over a non-amenable group, extending work of Gaboriau-Lyons. The proof uses an approximation to the random interlacement process by random multisets of geometrically-killed random walk paths. There are two applications: (1) the Gaboriau-Lyons problem for actions with positive Rokhlin entropy admits a positive solution, (2) for any non-amenable group, all Bernoulli shifts factor onto each other.
\end{abstract}

Keywords: random interlacement, von Neumann-Day problem, Gaboriau-Lyons, non-amenable groups, Bernoulli shifts

MSC:37A35

${ }^{*}$ supported in part by NSF grant DMS-1500389 


\section{Contents}

1 Introduction $\quad 3$

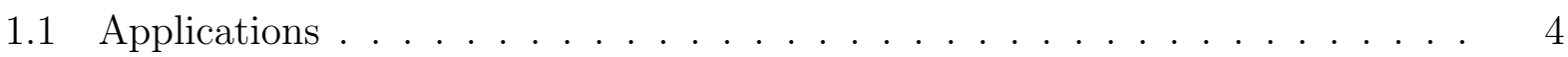

1.1.1 Positive entropy actions . . . . . . . . . . . . . . 4

1.1.2 Factors of Bernoulli shifts . . . . . . . . . . . . . 5

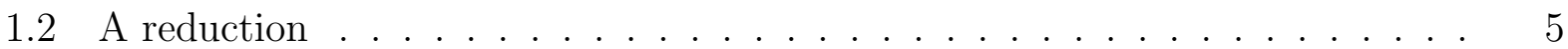

1.3 Finitary Random Interlacements . . . . . . . . . . . . . . . . . . 6

1.3.1 The big picture .......................... 6

1.3.2 An informal description . . . . . . . . . . . . . . 6

1.3.3 A brief sketch ..................... 8

1.4 Connections with Random Interlacements . . . . . . . . . . . . . . 8

1.5 Related Literature . . . . . . . . . . . . . . . . . . . . . . . . . 9

2 Notation

3 Finitary random interlacements $\quad 10$

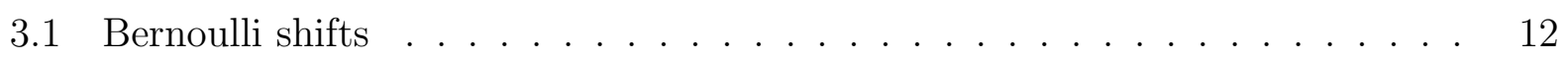

4 The local picture $\quad 14$

4.1 The cluster at the origin ......................... 16

5 Random walks on non-amenable graphs $\quad 17$

6 A truncated growth process $\quad 18$

6.1 At least one infinite cluster . . . . . . . . . . . . . . . 22

\begin{tabular}{ll|l|}
7 & Infinitely many infinite clusters & 24 \\
\hline
\end{tabular}

7.1 A random colored forest . . . . . . . . . . . . . . . . . 24

7.2 The random map into $G \ldots \ldots \ldots$. . . . . . . . . . . . . . . . . . . . . . . .

7.3 Branching random walk . . . . . . . . . . . . . . . 26

8 Infinitely many ends and indistinguishability 29 
9 Proof of Theorem $1.1 \quad 29$

9.1 Cost and treeability . . . . . . . . . . . . . . . . . . 30

9.2 Strong solidity . . . . . . . . . . . . . . . . . . . . . 31

9.3 The final step . . . . . . . . . . . . . . . . . . . . . 32

10 Applications $\quad 34$

10.1 Actions with positive Rokhlin entropy . . . . . . . . . . . . . 34

10.2 Factors of Bernoulli shifts . . . . . . . . . . . . . . . 35

\begin{tabular}{|l|l|} 
A Convergence to RI & 36 \\
\hline
\end{tabular}

\section{Introduction}

Amenability is inherited by subgroups and nonabelian free groups are non-amenable. These observations lead to the von Neumann-Day problem: does every non-amenable group contain a nonabelian free group? It was disproven by Ol'shankii [Ol'91]. However, the analogous problem for measured equivalence relations remains open. This article focuses on the special case of orbit-equivalence relations arising from free actions of countable groups:

Question 1 (Gaboriau-Lyons GL09]). Suppose $\Gamma$ is a countable group, $(X, \mathcal{B}, \mu)$ a standard probability space and $\Gamma \curvearrowright(X, \mathcal{B}, \mu)$ is an essentially free, ergodic, measure-preserving action. If $\Gamma$ is non-amenable then does there necessarily exist an essentially free, ergodic action $\mathbb{F}_{2} \curvearrowright(X, \mathcal{B}, \mu)$ of the rank 2 free group such that each orbit of the $\mathbb{F}_{2}$ action is contained in an orbit of the $\Gamma$ action (after neglecting a measure zero set)?

An action $\Gamma \curvearrowright(X, \mathcal{B}, \mu)$ is von Neumann-Day (vND) if either $\Gamma$ is amenable or the above question admits a positive answer. An action is weakly von Neumann-Day (weakly vND) if it satisfies the same condition as vND with the exception that the $\mathbb{F}_{2}$ action is allowed to be non-ergodic. So the Gaboriau-Lyons problem asks whether every essentially free, ergodic action of a countable group is vND1.

\footnotetext{
${ }^{1}$ The problem whether every ergodic measured equivalence relation is weakly vND was formulated by D. Gaboriau as Question 5.16 of his HDR thesis Gab02].
} 
Important progress was made by Gaboriau-Lyons [GL09]. Their result concerns Bernoulli shifts which are defined as follows. Let $K$ be a standard Borel space and $\kappa$ a Borel probability measure on $K$. Consider the product space $K^{\Gamma}=\{x: \Gamma \rightarrow K\}$ with the product sigmaalgebra and the product measure $\kappa^{\Gamma}$. The group $\Gamma$ acts on $K^{\Gamma}$ by shifting:

$$
(g x)(f)=x\left(g^{-1} f\right), \quad g, f \in \Gamma, x \in K^{\Gamma} .
$$

The action $\Gamma \curvearrowright(K, \kappa)^{\Gamma}$ is the Bernoulli shift over $\Gamma$ with base space $(K, \kappa)$.

If $\kappa$ is supported on a countable subset of $K$ then the Shannon entropy of $\kappa$ is defined by

$$
H(\kappa):=-\sum_{k \in K} \kappa(k) \log \kappa(k)
$$

If $\kappa$ is not supported on a countable set then $H(\kappa)$ is defined to be $+\infty$. In Bow10] and [KL11] it is shown that if $\Gamma$ is sofic then the Shannon entropy of the base space is a measure-conjugacy invariant. In [Sew18] Seward proved that, for any countable group $\Gamma$, if two base spaces have the same Shannon entropy then the corresponding Bernoulli shifts are isomorphic. Special cases of this result were previously obtained by Ornstein [Orn70] (for $\Gamma=\mathbb{Z}$ ), Stepin Ste75] (for groups containing an infinite cyclic subgroup), Ornstein-Weiss [OW80] (for amenable groups), and the author [Bow12] (for all countable groups and base spaces of cardinality $>2$ ). All of the above works build on the fundamental work of Ornstein Orn70.

Gaboriau and Lyons proved that for every non-amenable group $\Gamma$ there exists some probability space $(K, \kappa)$ such that the Bernoulli shift $\Gamma \curvearrowright(K, \kappa)^{\Gamma}$ is von Neumann-Day GL09] (see also the expository article [Hou12] for further applications). The main result of this paper is that the Gaboriau-Lyons Theorem holds for all nontrivial $(K, \kappa)$.

Theorem 1.1. Let $\Gamma$ be a countable non-amenable group and $(K, \kappa)$ a nontrivial probability space. Then the Bernoulli shift $\Gamma \curvearrowright(K, \kappa)^{\Gamma}$ is von Neumann-Day.

\subsection{Applications}

\subsubsection{Positive entropy actions}

In spectacular recent work, Seward generalized Sinai's Factor Theorem to all countable groups [Sew15]. Combined with Theorem 1.1, this implies: 
Corollary 1.2. Let $\Gamma$ be a countable non-amenable group and $\Gamma \curvearrowright(X, \mu)$ a probabilitymeasure-preserving ergodic essentially free action with positive Rokhlin entropy. Then this action is weakly von Neumann-Day.

The definition of Rokhlin entropy and further details are explained in \$10.1. I conjecture that the actions in Corollary 1.2 are actually vND.

\subsubsection{Factors of Bernoulli shifts}

An action $\Gamma \curvearrowright(X, \mu)$ factors onto another action $\Gamma \curvearrowright(Y, \nu)$ if there is a $\Gamma$-equivariant measurable map $\Phi: X \rightarrow Y$ with $\nu=\mu \circ \Phi^{-1}$. Two actions are weakly isomorphic if they factor onto each other.

If $\Gamma$ is amenable then the entropy of a factor action is bounded above by the entropy of the source. This is false for non-amenable groups. In fact Ornstein and Weiss exhibited by explicit example that the 2-shift over the rank 2 free group $\mathbb{F}_{2}$ factors onto the 4-shift [OW87]. In Bal05], K. Ball generalized this example to show that if $\Gamma$ is any non-amenable group then there is some Bernoulli shift $\Gamma \curvearrowright(K, \kappa)$ with $K$ finite that factors onto all Bernoulli shifts over $\Gamma$. In Bow11] I proved that if $\Gamma$ contains a non-abelian free group then all Bernoulli shifts over $\Gamma$ are weakly isomorphic. The techniques of these papers combined with [Sew18] and Theorem 1.1 prove:

Corollary 1.3. Let $\Gamma$ be any countable non-amenable group. Then all Bernoulli shifts over $\Gamma$ are weakly isomorphic.

Seward's Factor Theorem [Sew15] and Corollary 1.3 imply:

Corollary 1.4. Let $\Gamma$ be a countable non-amenable group and $\Gamma \curvearrowright(X, \mu)$ a probabilitymeasure-preserving, ergodic, essentially free action with positive Rokhlin entropy. Then this action factors onto all Bernoulli shifts over $\Gamma$.

\subsection{A reduction}

Gaboriau-Lyons GL09] uses the theory of cost to reduce their theorem to showing that a certain random subgraph of a Cayley graph of $\Gamma$ (whose law is measurably conjugate to 
a Bernoulli shift) is such that it has infinitely many infinite connected components with infinitely many ends a.s. Moreover it has indistinguishable infinite clusters in the sense of Lyons-Schramm [LS99]. We will use the same reduction. However, since the random graph we use is obtained from a nontrivial factor of a Bernoulli shift, this only shows the weak von Neumann-Day property. To finish the proof, we use the Chifan-Ioana Theorem that Bernoulli shifts over non-amenable groups are solidly ergodic [CI10].

\subsection{Finitary Random Interlacements}

\subsubsection{The big picture}

Theorem 1.1 is obtained by studying random multisets of finite random walks on a fixed Cayley graph of $\Gamma$. These random multisets are called Finitary Random Interlacements (FRI). The union of the random walk paths in an FRI is a random subgraph that satisfies the aforementioned reduction when certain parameter bounds hold. Moreover, the action of $\Gamma$ on the measure-space defining the FRI is measurably conjugate to a Bernoulli shift.

The FRI is a variant of the random interlacement process (RI) which is a random multiset of bi-infinite random walk paths introduced by Sznitman [Szn10]. The paper [TT13] proves that, for non-amenable graphs, the RI at low intensity has infinitely many infinite components. It is unknown whether the RI at low-intensity is a factor of a low-entropy Bernoulli shift. If it were true, it could be used to give another proof of Theorem 1.1, It was this observation that led to the approach of this paper. Moreover, we use essentially the same proof as in [TT13] to show that a low-intensity FRI has infinitely many infinite components whenever it has infinite components.

\subsubsection{An informal description}

The FRI is defined on a locally finite graph $G=(V, E)$. There are two parameters, $u, T>0$ called the intensity and average stopping time. To each vertex $x \in V$ is associated a Poisson random variable $\mathbf{N}_{x}$ with mean $u \operatorname{deg}_{x} /(T+1)$ where $\operatorname{deg}_{x}$ is the degree of $x$. Informally, we think of $\mathbf{N}_{x}$ as the number of frogs that live on vertex $x$ at time 0 . Each frog has a coin that lands on heads with probability $p=\frac{T}{T+1}$. At time 0 , a frog flips her coin. If it lands on heads, the frog moves to a uniformly random neighboring vertex. It repeats this 
operation until the coin lands on tails at which point the frog stops forever. So each frog performs a simple random walk for $t$ steps where $t+1$ is a geometric random variable with mean $T+1$. The FRI is the random multiset of these finite random walk paths.

It might help the reader's intuition to know that the expected number of walks of the FRI that traverse a fixed vertex $x$ is $u \operatorname{deg}_{x} P_{x}^{(T)}\left(\tilde{\mathrm{H}}_{x}=\infty\right)$ where $P_{x}^{(T)}\left(\tilde{\mathrm{H}}_{x}=\infty\right)$ is the probability that the aforementioned geometrically-killed random walk started at $x$ does not return to $x$. In particular, if $G$ is vertex-transitive then this expected number of walks is proportional to $u$.

To be more precise, let $\mathcal{W}[0, \infty)$ denote the collection of all finite walks $w: D_{w} \rightarrow V$ where $D_{w}=[0, \operatorname{len}(w)] \cap \mathbb{N}$ for some $\operatorname{len}(w) \in \mathbb{N}$ called the length of $w$. By convention $\mathbb{N}=\{0,1,2, \ldots\}$. Then $\mathcal{W}[0, \infty)$ is a countable set because $V$ is countable. Let $\Omega^{[0, \infty)}$ be the set of all locally finite $\mathbb{N} \cup\{\infty\}$-valued measures on $\mathcal{W}[0, \infty)$. We can identify $\Omega^{[0, \infty)}$ with a subspace of the space of all functions from $\mathcal{W}[0, \infty)$ to $\mathbb{N}$ endowed with the topology of pointwise convergence. Finally the law of the FRI is a probability measure $\mathbb{P}_{u, T}$ on $\Omega^{[0, \infty)}$.

Given $\omega \in \Omega^{[0, \infty)}$, let $\mathcal{E}_{\omega} \subset E$ denote the collection of edges crossed by at least one walk in the support of $\omega$. Recall that a cluster is a connected component. Our main result is:

Theorem 1.5. Let $\Gamma$ be a non-amenable countable group with a finite generating set $S \subset \Gamma$. Let $G=(V, E)$ denote the associated Cayley graph. Then:

1. for every $\epsilon>0$ there exists $u_{0}>0$ such that if $0<u<u_{0}$ and $T>0$ then the action $\Gamma \curvearrowright\left(\Omega^{[0, \infty)}, \mathbb{P}_{u, T}\right)$ is measurably conjugate to a Bernoulli shift over $\Gamma$ with base entropy $<\epsilon$;

2. for every $u>0$ there exists $T_{u}$ such that if $T>T_{u}$ then for $\mathbb{P}_{u, T}$-almost every $\omega$, the subgraph $\left(V, \mathcal{E}_{\omega}\right)$ has infinite clusters;

3. there exists $u_{c}>0$ such that if $0<u<u_{c}$ and $T>T_{u}$ then for $\mathbb{P}_{u, T}$-almost every $\omega$, the subgraph $\left(V, \mathcal{E}_{\omega}\right)$ has infinitely many infinite clusters;

4. for any $u, T>0$, if $\left(V, \mathcal{E}_{\omega}\right)$ has infinitely many infinite clusters $\mathbb{P}_{u, T}$-a.s. then each infinite cluster has infinitely many ends $\mathbb{P}_{u, T}$-a.s. Moreover if $\boldsymbol{\omega} \sim \mathbb{P}_{u, T}$ then the random subgraph $\left(V, \mathcal{E}_{\boldsymbol{\omega}}\right)$ has indistinguishable infinite clusters in the sense of LyonsSchramm [LS99]. 
Question 2. Does item (2) hold when $\Gamma$ is amenable and simple random walk on its Cayley graph is transient? In particular, does it hold on $\mathbb{Z}^{d}$ ?

Remark 1. Parts (2) and (3) of the theorem can be extended to all graphs $G$ that are bounded degree, connected and non-amenable. The conclusion of item (4) holds a.s. if $G$ is a unimodular network. For clarity's sake, the theorem is proven first for Cayley graphs, and then it is explained how minor changes give this generalization.

\subsubsection{A brief sketch}

Theorem 1.5 is proven in several steps. The first statement follows from direct entropy computation. To prove the existence of infinite clusters, it is shown that the cluster containing the origin stochastically dominates a certain growth process which is analyzed with a second moment argument. To prove that infinite clusters and low-intensity implies the existence of infinitely many infinite components, it is shown that the subgraph of $\left(V, \mathcal{E}_{\omega}\right)$ containing the origin is stochastically dominated by a branching random walk with $1+O(u)$ mean offspring distribution. This is similar to the way Benjamini-Schramm obtained bounds on the non-uniqueness phase in Bernoulli percolation [BS11] and the way Teixeira-Tykesson proved that low-intensity Random Interlacements have infinitely many components in non-amenable graphs [TT13].

Lastly, it is observed if $\boldsymbol{\omega} \sim \mathbb{P}_{u, T}$ then the random subgraph $\left(V, \mathcal{E}_{\boldsymbol{\omega}}\right)$ is insertion-tolerant. So by [LS99], whenever $G$ is vertex-transitive and $\left(V, \mathcal{E}_{\boldsymbol{\omega}}\right)$ has infinitely many infinite clusters, the infinite clusters are indistinguishable and have infinitely many ends a.s.

\subsection{Connections with Random Interlacements}

Random Interlacements (RI) are random multisets of bi-infinite random walk paths on a locally finite graph $G=(V, E)$. They were introduced by Sznitman [Szn10] where the focus is on the connectedness properties of the complement in the case when $G$ is a cubic lattice. See also [DRS14] for a general introduction and [Tei09] for a definition of RI on general weighted transient graphs.

The 'local picture' of Finitary Random Interlacements is similar to standard constructions

of Random Interlacements, which I learned from [TT13]. In the appendix it is shown that 
the FRI process converges in distribution to the RI as $T \rightarrow \infty$. This result is not needed for any of the other results; we have included it only to justify the name FRI.

\subsection{Related Literature}

There is an excellent Bourbaki article on the Gaboriau-Lyons Theorem and its applications to measurable group theory [Hou12].

As far as I am aware, before the Gaboriau-Lyons Theorem there was only one technique for proving the existence of a free subgroup (measurable or otherwise): the ping-pong lemma used in the proof of the Tit's Alternative [Tit72]. That idea was used in [Bow18] to prove that measured equivalence relations that act properly on a bundle of hyperbolic spaces are von Neumann-Day.

The Gaboriau-Lyons Theorem was extended in [BHI18] to class-bijective extensions of measured equivalence relations. The techniques of that paper can be combined with this article to strengthen the main result of [BHI18] so that it holds for all Bernoulli shifts.

In Kun13, Gabor Kun obtains a Lipschitz version of the Gaboriau-Lyons Theorem. I do not know if there exists a Lipschitz version of Theorem 1.1 .

Acknowledgements. I am deeply grateful to Itai Benjamini for a series of email conversations on random interlacements which initiated this approach. Also thanks to the authors of [TT13] from which I learned most of what I know about random interlacements. Thanks also to the anonymous referee for suggestions that greatly simplified several proofs.

\section{Notation}

Throughout this paper, $\mathbb{N}=\{0,1,2, \ldots\}$. The notation $A \Subset B$ means that $A$ is a finite subset of $B$.

We use boldface to denote random variables. For example, suppose $\mathbb{P}$ is a probability measure on a space $\Omega$. Then $\omega \in \Omega$ denotes an element of $\Omega$ while $\boldsymbol{\omega} \sim \mathbb{P}$ denotes a random variable taking values in $\Omega$ with law $\mathbb{P}$. In other words, for any measurable event $E \subset \Omega$, $\mathbb{P}(\boldsymbol{\omega} \in E)=\mathbb{P}(E)$. Also $\mathbf{N} \sim \operatorname{Poisson}(m)$ indicates that $\mathbf{N}$ is a Poisson random variable with mean $m$ and $\mathbf{N}+1 \sim \operatorname{Geom}(T)$ indicates that $\mathbf{N}+1$ is a geometric random variable 
with expected value $T$. Finally, $\mathbf{N}_{1} \sim \mathbf{N}_{2}$ means that $\mathbf{N}_{1}$ and $\mathbf{N}_{2}$ are identically distributed random variables. If $\omega \in \Omega$ then we may write $\mathbb{P}(\omega)$ instead of $\mathbb{P}(\{\omega\})$ for simplicity.

For any measure $\kappa$ on a set $X$ and any measurable map $\Phi: X \rightarrow Y$ the pushforward measure $\Phi_{*} \kappa$ on $Y$ is defined by $\Phi_{*} \kappa=\kappa \circ \Phi^{-1}$.

Let $(X, \mathcal{B}, \mu)$ be a Borel space with a sigma-finite measure $\mu$. The Poisson point process with intensity measure $\mu$ is a random $\mathbb{N} \cup\{\infty\}$-valued measure $\boldsymbol{\omega}$ on $X$ satisfying

- if $A_{1}, A_{2}, \ldots \subset X$ are disjoint measurable sets then the restrictions $\left\{\boldsymbol{\omega} \uparrow A_{i}\right\}_{i}$ are jointly independent;

- for any measurable $A \subset X$ with $\mu(A)<\infty, \boldsymbol{\omega}(A)$ is a Poisson random variable with mean $\mu(A)$.

Thus if $\Omega$ is the set of all $\mathbb{N} \cup\{\infty\}$-valued measures on $X$ endowed with the sigma-algebra generated by the functions $\omega \mapsto \omega(A)$ (for $A \subset X$ measurable) then the law of the Poisson point process $\boldsymbol{\omega}$ is the unique probability measure $\mathbb{P}$ on $\Omega$ satisfying

1. if $A_{1}, A_{2}, \ldots \subset X$ are disjoint measurable sets and $\mathcal{A}_{i}$ is the sigma-algebra on $\Omega$ defined by restricting to $A_{i}$ then the sigma-algebras $\mathcal{A}_{1}, \mathcal{A}_{2}, \ldots$ are jointly independent with respect to $\mathbb{P}$,

2. for any measurable $A \subset X$ with $\mu(A)<\infty$ and $n \in \mathbb{N}$,

$$
\mathbb{P}(\{\omega \in \Omega: \omega(A)=n\})=\exp (-\mu(A)) \frac{\mu(A)^{n}}{n !} .
$$

\section{$3 \quad$ Finitary random interlacements}

We will use notation as in [Hut18, $\S 3.2]$. Let $G=(V, E)$ be a locally finite connected graph on countably many vertices. For each $-\infty \leq m \leq n \leq \infty$, let $L(m, n)$ be the graph with vertex set $\{i \in \mathbb{Z}: m \leq i \leq n\}$ and edge set $\{(i, i+1): m \leq i \leq n-1\}$. Let $\mathcal{W}[m, n]$ be the set of multigraph homomorphisms $w: L(m, n) \rightarrow G$ such that the pre-image of each vertex in $G$ is finite, and let $\mathcal{W}, \mathcal{W}[0, \infty)$ be the unions

$$
\begin{aligned}
\mathcal{W} & =\bigcup_{-\infty \leq m \leq n \leq \infty} \mathcal{W}[m, n] \\
\mathcal{W}[0, \infty) & =\bigcup_{0 \leq n<\infty} \mathcal{W}[0, n] .
\end{aligned}
$$


For $w \in \mathcal{W}[m, n]$, let len $(w)=n-m$ denote its length and $D_{w}=\{m, \ldots, n\}$ its domain.

Definition 1. For $x \in V$ and $0 \leq n \leq \infty$, let $P_{x}^{n}$ be the law of the simple random walk started from $x$ and stopped at time $n$. More precisely, $P_{x}^{n}$ is the Borel probability measure on $\mathcal{W}$ satisfying

$$
P_{x}^{n}(w)=\left\{\begin{array}{cl}
\prod_{k=0}^{n-1} \operatorname{deg}_{w(k)}^{-1} & \text { if } w \in \mathcal{W}[0, n] \text { and } w(0)=x \\
0 & \text { otherwise }
\end{array}\right.
$$

For simplicity, we write $P_{x}$ for $P_{x}^{\infty}$. Let

$$
P_{x}^{(T)}=\left(\frac{1}{T+1}\right) \sum_{n \in \mathbb{N}}\left(\frac{T}{T+1}\right)^{n} P_{x}^{n}
$$

denote the geometrization of the measures $P_{x}^{n}$. Thus $P_{x}^{(T)}$ is the law of the simple random walk started from $x$ with random stopping time $\sim \operatorname{Geom}(T+1)-1$.

A counting measure is a measure taking values in $\mathbb{N} \cup\{\infty\}$. Given $K \subset V$, let $\mathcal{W}_{K}$ be the set of all walks $w \in \mathcal{W}$ such that $w(i) \in K$ for some $i$. A measure $\omega$ on $\mathcal{W}$ is locally finite if $\omega\left(W_{K}\right)<\infty$ for every finite $K \subset V$.

Since $\mathcal{W}[0, \infty)$ is countable, we give it the $\sigma$-algebra of all subsets. Let $\Omega^{[0, \infty)}$ denote the space of all locally finite counting measures $\omega$ on $\mathcal{W}[0, \infty)$. We can think of $\Omega^{[0, \infty)}$ as a subset of the set of all functions from $\mathcal{W}[0, \infty)$ to $\mathbb{N}$ (which is denoted by $\mathbb{N}^{\mathcal{W}[0, \infty)}$ ). We give $\mathbb{N}^{\mathcal{W}[0, \infty}$ ) the topology of pointwise convergence under which it is a Polish space. Because $\Omega^{[0, \infty)}$ is a $G_{\delta}$ subset of $\mathbb{N}^{\mathcal{W}[0, \infty)}, \Omega^{[0, \infty)}$ is also Polish.

Definition 2 (Finitary random interlacements). For $0<T<\infty$, let $\nu^{(T)}$ be the measure on $\mathcal{W}$ defined by

$$
\nu^{(T)}=\sum_{x \in V} \frac{\operatorname{deg}_{x}}{T+1} P_{x}^{(T)} .
$$

This measure is infinite if $V$ is infinite. For $0<u, T<\infty$, the finitary random interlacement, typically denoted by $\boldsymbol{\omega}$, is the Poisson point process on $\mathcal{W}$ with intensity measure $u \nu^{(T)}$. The law of $\boldsymbol{\omega}$ is a probability measure on $\Omega^{[0, \infty)}$ denoted by $\mathbb{P}_{u, T}$. To be precise, this means that for any $w_{1}, \ldots, w_{k} \in \mathcal{W}[0, \infty)$ and natural numbers $n_{1}, \ldots, n_{k}$,

$$
\begin{aligned}
\mathbb{P}_{u, T}\left(\left\{\omega \in \Omega^{[0, \infty)}: \omega\left(w_{i}\right)=n_{i} \forall 1 \leq i \leq k\right\}\right) & =\mathbb{P}_{u, T}\left(\boldsymbol{\omega}\left(w_{i}\right)=n_{i} \forall 1 \leq i \leq k\right) \\
& =\prod_{i=1}^{k} \exp \left(-u \nu^{(T)}\left(w_{i}\right)\right) \frac{\left(u \nu^{(T)}\left(w_{i}\right)\right)^{n_{i}}}{n_{i} !}
\end{aligned}
$$


In other words, $\boldsymbol{\omega}$ is a random element of $\Omega^{[0, \infty)}$ whose law is uniquely determined by:

1. the family of random variables $\{\boldsymbol{\omega}(w): w \in \mathcal{W}[0, \infty)\}$ is jointly independent;

2. for each $w \in \mathcal{W}[0, \infty), \boldsymbol{\omega}(w)$ is a Poisson random variable with mean $u \nu^{(T)}(w)$.

\subsection{Bernoulli shifts}

This section proves part (1) of Theorem 1.5. So let $\Gamma$ be a countable group with a finite symmetric generating set $S$ and let $G=(V, E)$ be the associated Cayley graph. To be precise, $V=\Gamma$ and $E$ is the set of all unordered pairs $\{g, g s\}$ for $g \in \Gamma, s \in S$. Because $\Gamma$ acts on $V$ by left-translation, $\Gamma$ also acts on $\mathcal{W}[0, \infty)$ by

$$
(g w)(n)=g w(n) \quad \forall g \in \Gamma, w \in \mathcal{W}[0, \infty), n \in D_{w}
$$

and $\Gamma$ acts on $\Omega^{[0, \infty)}$ by $(g \omega)(E)=\omega\left(g^{-1} E\right)$. The group $\Gamma$ is not required to be non-amenable for the next result.

Theorem 3.1. With notation as above, for every $\epsilon>0$ there exists $u_{\epsilon}>0$ such that if $u<u_{\epsilon}$ then $\Gamma \curvearrowright\left(\Omega^{[0, \infty)}, \mathbb{P}_{u, T}\right)$ is measurably conjugate to a Bernoulli shift with base entropy at most $\epsilon$.

Proof. Let $\mathcal{W}_{g}[0, \infty) \subset \mathcal{W}[0, \infty)$ be the set of all finite walks on $G$ that start at $g \in V=\Gamma$. Let $\Omega_{g}^{[0, \infty)}$ be the set of all $\omega \in \Omega^{[0, \infty)}$ such that $\omega$ is finite and supported on $\mathcal{W}_{g}[0, \infty)$. Both $\mathcal{W}_{g}[0, \infty)$ and $\Omega_{g}^{[0, \infty)}$ are countable sets.

Let $e \in \Gamma$ denote the identity element. Define a probability measure on $\Omega_{e}^{[0, \infty)}$ as follows. First let $\mathbf{N}$ be a Poisson random variable with mean $u|S| /(T+1)$. For each $1 \leq i \leq \mathbf{N}$, let $\mathbf{w}_{i} \in \mathcal{W}_{e}[0, \infty)$ be a random walk with law $P_{e}^{(T)}$. Now let $\boldsymbol{\omega}=\sum_{i=1}^{\mathbf{N}} \delta_{\mathbf{w}_{i}}$. Let $\eta$ be the law of $\boldsymbol{\omega}$. So $\eta$ is a probability measure on $\Omega_{e}^{[0, \infty)}$.

For $\omega \in \Omega_{e}^{[0, \infty)}$ and $g \in \Gamma$, define

$$
g * \omega \in \Omega_{g}^{[0, \infty)} \text { by } g * \omega(\{w\})=\omega\left(\left\{g^{-1} w\right\}\right) .
$$

We claim that $\Gamma \curvearrowright\left(\Omega^{[0, \infty)}, \mathbb{P}_{u, T}\right)$ is measurably conjugate to the Bernoulli shift $\Gamma \curvearrowright\left(\Omega_{e}^{[0, \infty)}, \eta\right)^{\Gamma}$. To see this, denote an arbitrary element $\xi \in\left(\Omega_{e}^{[0, \infty)}\right)^{\Gamma}$ by $\xi=\left(\xi_{g}\right)_{g \in \Gamma}$ and define

$$
\Phi:\left(\Omega_{e}^{[0, \infty)}\right)^{\Gamma} \rightarrow \Omega^{[0, \infty)} \text { by } \Phi(\xi)=\sum_{g \in \Gamma} g * \xi_{g} .
$$


To check that $\Phi$ is $\Gamma$-equivariant, let $h \in \Gamma$ and $w \in \mathcal{W}_{g}[0, \infty)$. Then

$$
\begin{aligned}
\Phi(h \xi)(\{w\}) & =g *(h \xi)_{g}(\{w\})=(h \xi)_{g}\left(\left\{g^{-1} w\right\}\right)=\xi_{h^{-1} g}\left(\left\{g^{-1} w\right\}\right)=\xi_{h^{-1} g}\left(\left\{g^{-1} h h^{-1} w\right\}\right) \\
& =\left(h^{-1} g\right) * \xi_{h^{-1} g}\left(\left\{h^{-1} w\right\}\right)=\Phi(\xi)\left(\left\{h^{-1} w\right\}\right)=h \Phi(\xi)(\{w\}) .
\end{aligned}
$$

Moreover $\Phi$ is invertible with inverse defined by

$$
\Phi^{-1}(\omega)_{g}(\{w\})=\omega(\{g w\}) \quad \forall g \in \Gamma, w \in \mathcal{W}_{e}[0, \infty)
$$

By construction of $\eta, \Phi_{*} \eta^{\Gamma}=\mathbb{P}_{u, T}$. This proves the claim.

Next we turn towards the entropy computation. If $\mathbf{X}$ is any random variable taking values in a countable set $A$ then the Shannon entropy of $\mathbf{X}$ is

$$
H(\mathbf{X}):=\sum_{a \in A}-\operatorname{Prob}(\mathbf{X}=a) \log (\operatorname{Prob}(\mathbf{X}=a))
$$

If $\mathbf{Y}$ is another random variable taking values in a countable set $B$ then $H(\mathbf{X}, \mathbf{Y})$ is the Shannon entropy of the join $\mathbf{X} \vee \mathbf{Y}$ which takes values in $A \times B$. We will assume standard facts about entropy and relative entropy that can be found in [CT06] for example. We let $\mathbb{E}$ denote expected value.

Let $\mathbf{N}, \mathbf{w}_{1}, \ldots, \mathbf{w}_{\mathbf{N}}$ be as above. Let $\mathbf{L}_{i}=\operatorname{len}\left(\mathbf{w}_{i}\right)$ be the length of $\mathbf{w}_{i}$. Then

$$
\begin{aligned}
H(\eta) & \leq H\left(\mathbf{N}, \mathbf{L}_{1}, \ldots, \mathbf{L}_{\mathbf{N}}, \mathbf{w}_{1}, \ldots, \mathbf{w}_{\mathbf{N}}\right) \\
& =H(\mathbf{N})+H\left(\mathbf{L}_{1}, \ldots, \mathbf{L}_{\mathbf{N}} \mid \mathbf{N}\right)+H\left(\mathbf{w}_{1}, \ldots, \mathbf{w}_{\mathbf{N}} \mid \mathbf{N}, \mathbf{L}_{1}, \ldots, \mathbf{L}_{\mathbf{N}}\right)
\end{aligned}
$$

Next we estimate each of the three terms on the right hand side.

Because $\mathbf{N}$ is a Poisson random variable with mean $u|S| /(T+1)=O(u), H(\mathbf{N})=O(u)$. Because the $\mathbf{L}_{i}$ 's are i.i.d. and independent of $\mathbf{N}$,

$$
H\left(\mathbf{L}_{1}, \ldots, \mathbf{L}_{\mathbf{N}} \mid \mathbf{N}\right)=\mathbb{E}[\mathbf{N}] H\left(\mathbf{L}_{1}\right)=\frac{u|S|}{T+1} H\left(\mathbf{L}_{1}\right)
$$

Since $\mathbf{L}_{1}+1$ is a geometric random variable with mean $T+1$,

$$
H\left(\mathbf{L}_{1}\right)=(T+1) H\left(\frac{1}{T+1}, \frac{T}{T+1}\right) \leq \log (2)(T+1)
$$

(where $H(x, 1-x):=-x \log x-(1-x) \log (1-x))$. Thus

$$
H\left(\mathbf{L}_{1}, \ldots, \mathbf{L}_{\mathbf{N}} \mid \mathbf{N}\right)=O(u)
$$


Because the $\mathbf{w}_{i}$ 's are jointly independent and $\mathbf{w}_{i}$ depends only on $\mathbf{L}_{i}$,

$$
H\left(\mathbf{w}_{1}, \ldots, \mathbf{w}_{\mathbf{N}} \mid \mathbf{N}, \mathbf{L}_{1}, \ldots, \mathbf{L}_{\mathbf{N}}\right)=\mathbb{E}[\mathbf{N}] H\left(\mathbf{w}_{1} \mid \mathbf{L}_{1}\right)=\frac{u|S|}{T+1} H\left(\mathbf{w}_{1} \mid \mathbf{L}_{1}\right) .
$$

If $\mathbf{L}_{1}=k$ then $\mathbf{w}_{1}$ is uniformly distributed on a set of cardinality $|S|^{k}$. So $H\left(\mathbf{w}_{1} \mid \mathbf{L}_{1}\right)=$ $\mathbb{E}\left[\mathbf{L}_{1}\right] \log |S|=T \log |S|$. So

$$
H\left(\mathbf{w}_{1}, \ldots, \mathbf{w}_{\mathbf{N}} \mid \mathbf{N}, \mathbf{L}_{1}, \ldots, \mathbf{L}_{\mathbf{N}}\right)=O(u)
$$

The previous three estimates imply $H(\eta)=O(u)$ as required.

\section{The local picture}

Let $K \Subset L \Subset V$ be finite subsets of $V$. Let $\mathcal{W}_{K} \subset \mathcal{W}$ be the set of all walks $w$ that visit $K$ at least once (i.e., $w(i) \in K$ for some $i$ ). Define $\mathcal{W}_{L}$ similarly. The goal of the next result is to describe the distribution of $\boldsymbol{\omega} \uparrow\left(\mathcal{W}_{L} \backslash \mathcal{W}_{K}\right)$ where $\boldsymbol{\omega} \sim \mathbb{P}_{u, T}$. This will be used later to describe the cluster at the origin by a growth process.

Definition 3. Define the $K$-hitting times for $w \in \mathcal{W}[m, n]$ by

$$
\begin{gathered}
H_{K}(w)=\inf \{t \in \mathbb{N}: w(t) \in K\} \in \mathbb{N} \cup\{\infty\} \\
\tilde{\mathrm{H}}_{K}(w)=\inf \{t \geq 1: w(t) \in K\} \in\{1,2, \ldots\} \cup\{\infty\} .
\end{gathered}
$$

By convention the infimum of the empty set is $+\infty$. If $K=\{x\}$ is a singleton then write $H_{x}(\cdot)$ and $\tilde{\mathrm{H}}_{x}(\cdot)$ instead of $H_{\{x\}}(\cdot), \tilde{\mathrm{H}}_{\{x\}}(\cdot)$ for simplicity.

Let $\mathcal{W}^{(2)}$ be the set of all pairs of walks $(a, b) \in \mathcal{W}[0, \infty) \times \mathcal{W}[0, \infty)$ such that $a(0)=b(0)$. For $x \in L \backslash K$, let $\zeta_{x}^{(T)}$ be the measure on $\mathcal{W}[0, \infty)_{x} \times \mathcal{W}[0, \infty)_{x} \subset \mathcal{W}^{(2)}$ given by

$$
\zeta_{x}^{(T)}(\{(a, b)\})=1_{\tilde{\mathrm{H}}_{L}(a)=\infty} P_{x}^{(T)}(\{a\}) \operatorname{deg}_{x} 1_{H_{K}(b)=\infty} P_{x}^{(T)}(\{b\}) .
$$

Define a measure $Q_{L, K}^{(T)}$ on $\mathcal{W}^{(2)}$ by

$$
Q_{L, K}^{(T)}=\sum_{x \in L \backslash K} \zeta_{x}^{(T)}
$$

The concatenation map is defined by

Con $: \mathcal{W}^{(2)} \rightarrow \mathcal{W}[0, \infty), \quad \operatorname{Con}(a, b)=(a(\operatorname{len}(a)), a(\operatorname{len}(a)-1), \ldots, a(0), b(1), \ldots, b(\operatorname{len}(b)))$.

For example, this means that $\operatorname{Con}(a, b)(0)=a(\operatorname{len}(a))$. 
Proposition 4.1. For $0<u, T<\infty$, let $\boldsymbol{\omega} \sim \mathbb{P}_{u, T}$ and $K \Subset L \Subset V$ be finite sets. Then the restriction $\boldsymbol{\omega} \uparrow\left(\mathcal{W}_{L} \backslash \mathcal{W}_{K}\right)$ is a Poisson point process with intensity measure $u \operatorname{Con}_{*} Q_{L, K}^{(T)}$.

Proof. By definition, the restriction $\boldsymbol{\omega} \uparrow\left(\mathcal{W}_{L} \backslash \mathcal{W}_{K}\right)$ is a Poisson point process with intensity measure equal to the restriction of $u \nu^{(T)}$ to $\mathcal{W}_{L} \backslash \mathcal{W}_{K}$. So it suffices to prove that if $w \in$ $\mathcal{W}_{L} \backslash \mathcal{W}_{K}$ then $\nu^{(T)}(\{w\})=\operatorname{Con}_{*} Q_{L, K}^{(T)}(\{w\})$. Let $t \geq 0$ be the smallest number such that $w(t) \in L$. Define walks $a, b$ by

$$
a=(w(t), w(t-1), \ldots, w(0)), \quad b=(w(t), w(t+1), \ldots, w(\operatorname{len}(w))) .
$$

Then $(a, b) \in \mathcal{W}^{(2)}$ is the unique pair such that $\operatorname{Con}(a, b)=w$ and $Q_{L, K}^{(T)}(\{(a, b)\})>0$. Indeed, if $\left(a^{\prime}, b^{\prime}\right) \in \mathcal{W}_{<\infty}^{(2)}$ is any pair with $\operatorname{Con}\left(a^{\prime}, b^{\prime}\right)=w$ then there is an $s \in[0, \operatorname{len}(w)] \cap \mathbb{N}$ such that

$$
a^{\prime}=(w(s), w(s-1), \ldots, w(0)), \quad b^{\prime}=(w(s), w(s+1), \ldots, w(\operatorname{len}(w))) .
$$

Suppose $Q_{L, K}^{(T)}\left(\left\{\left(a^{\prime}, b^{\prime}\right)\right\}\right)>0$. Then $w(s) \in L$. If $s \neq t$ then $\tilde{\mathrm{H}}_{L}\left(a^{\prime}\right) \leq s-t<\infty$. But this implies $Q_{L, K}^{(T)}\left(\left\{\left(a^{\prime}, b^{\prime}\right)\right\}\right)=0$, a contradiction.

Let $x=a(0)=b(0)$. Since $Q_{L, K}^{(T)}(\{(a, b)\})=\zeta_{x}(\{(a, b)\})$, it now suffices to prove $\nu^{(T)}(\{w\})=\zeta_{x}(\{(a, b)\})$. To simplify the computation, let $\lambda=\operatorname{len}(w), \alpha=\operatorname{len}(a), \beta=$ len $(b)$. Then

$$
\begin{aligned}
\nu^{(T)}(\{w\}) & =\frac{\operatorname{deg}_{w(0)}}{T+1} P_{w(0)}^{(T)}(\{w\})=\frac{\operatorname{deg}_{w(0)}}{(T+1)^{2}}\left(\frac{T}{T+1}\right)^{\lambda} \operatorname{deg}_{w(0)}^{-1} \cdots \operatorname{deg}_{w(\lambda-1)}^{-1} \\
& =\frac{1}{(T+1)^{2}}\left(\frac{T}{T+1}\right)^{\lambda} \operatorname{deg}_{w(1)}^{-1} \cdots \operatorname{deg}_{w(\lambda-1)}^{-1}
\end{aligned}
$$

On the other hand,

$$
\begin{aligned}
\zeta_{x}(\{(a, b)\}) & =P^{(T)}(\{a\}) \operatorname{deg}_{x} P^{(T)}(\{b\}) \\
& =\frac{1}{(T+1)^{2}}\left(\frac{T}{T+1}\right)^{\alpha+\beta} \operatorname{deg}_{a(0)}^{-1} \cdots \operatorname{deg}_{a(\alpha-1)}^{-1} \operatorname{deg}_{x} \operatorname{deg}_{b(0)}^{-1} \cdots \operatorname{deg}_{b(\beta-1)}^{-1} .
\end{aligned}
$$

The fact that $\nu^{(T)}(\{w\})=\zeta_{x}(\{(a, b)\})$ now follows from: $\lambda=\alpha+\beta, x=b(0)$ and

$$
(w(1), \ldots, w(\lambda-1))=(a(\alpha-1), \ldots, a(0), b(1), \ldots, b(\beta-1)) .
$$


Remark 2. An easy special case of the above result helps aid the intuition. Suppose $K=\emptyset$ and $L=\{x\}$ is a single vertex. Then Proposition 4.1 implies: if $\boldsymbol{\omega} \sim \mathbb{P}_{u, T}$ then the expected value of

$$
\boldsymbol{\omega}\left(\left\{w \in \mathcal{W}[0, \infty): H_{x}(w)<\infty\right\}\right)
$$

is $u \operatorname{deg}_{x} \mathbb{P}_{x}^{(T)}\left(\tilde{\mathrm{H}}_{x}=\infty\right)$. In particular, if $G$ is vertex transitive then this number is a constant multiple of $u$.

The next corollary provides a more intuitive description of the restriction $\boldsymbol{\omega} \uparrow\left(\mathcal{W}_{L} \backslash \mathcal{W}_{K}\right)$. It follows immediately from Proposition 4.1 .

Corollary 4.2. Let $\boldsymbol{\omega} \sim \mathbb{P}_{u, T}$ and $K \Subset L \Subset V$ be finite sets. For every $x \in L \backslash K$, let $\mathbf{N}_{x}$ be a Poisson random variable with mean $u \operatorname{deg}_{x}$. For $i=1, \ldots, \mathbf{N}_{x}$, choose a pair $\left(\mathbf{a}_{x, i}, \mathbf{b}_{x, i}\right) \in \mathcal{W}_{x}^{[0, \infty)} \times \mathcal{W}_{x}^{[0, \infty)}$ at random with law $P_{x}^{(T)} \times P_{x}^{(T)}$. Let $\mathbf{R}_{x} \subset\left\{1, \ldots, \mathbf{N}_{x}\right\}$ be the set of all $j$ such that $\tilde{\mathrm{H}}_{L}\left(\mathbf{a}_{x, j}\right)=\infty$ and $H_{K}\left(\mathbf{b}_{x, j}\right)=\infty$. Then

$$
\boldsymbol{\omega} \uparrow\left(\mathcal{W}_{L} \backslash \mathcal{W}_{K}\right) \sim \sum_{x \in L \backslash K} \sum_{j \in \mathbf{R}_{x}} \delta_{C o n\left(\mathbf{a}_{x, j}, \mathbf{b}_{x, j}\right)}
$$

where $\delta$ denotes the Dirac delta measure.

\subsection{The cluster at the origin}

This section describes the cluster at the origin in terms of a growth process.

Definition 4. If $\omega \in \Omega^{[0, \infty)}$ then let $\mathcal{V}_{\omega}=\mathcal{V}(\omega)$ be the set of vertices traversed by some walk in the support of $\omega$. Similarly, $\mathcal{E}_{\omega}=\mathcal{E}(\omega)$ is the set of edges $e \in E$ traversed by some walk in the support of $\omega$.

Fix $x \in V$. Given $\omega \in \Omega^{[0, \infty)}$, let $\omega_{1}^{x}=\omega \uparrow \mathcal{W}_{x}$ be the restriction of $\omega$ to $\mathcal{W}_{x}$ (where $\mathcal{W}_{x}=\mathcal{W}_{\{x\}} \subset \mathcal{W}$ is the set of walks that contain $\left.x\right)$. For $n \geq 2$, inductively define $\omega_{n}^{x}$ by $\omega_{n}^{x}=\omega^{x} \uparrow \mathcal{W}_{\mathcal{V}\left(\omega_{n-1}^{x}\right)}$. Finally, define $\omega_{\infty}^{x}=\sup _{n} \omega_{n}^{x}$. Then $\left(\mathcal{V}\left(\omega_{\infty}^{x}\right), \mathcal{E}\left(\omega_{\infty}^{x}\right)\right)$ is the $\omega$-cluster containing $x$. Let $\mathbb{P}_{u, T}^{x}$ denote the law of $\boldsymbol{\omega}_{\infty}^{x}$ where $\boldsymbol{\omega} \sim \mathbb{P}_{u, T}$. So $\mathbb{P}_{u, T}^{x}$ is a probability measure on $\Omega^{[0, \infty)}$.

Proposition 4.3. Let $\boldsymbol{\omega} \sim \mathbb{P}_{u, T}$ and $n \geq 0$. Then $\boldsymbol{\omega}_{n+1}^{x}$ conditioned on $\mathcal{V}\left(\boldsymbol{\omega}_{n}\right)$ and $\mathcal{V}\left(\boldsymbol{\omega}_{n-1}\right)$ is a Poisson point process with intensity measure $u \operatorname{Con}_{*} Q_{\mathcal{V}\left(\boldsymbol{\omega}_{n}\right), \mathcal{V}\left(\boldsymbol{\omega}_{n-1}\right)}^{(T)}$.

Proof. This follows from Proposition 4.1 and the Poisson nature of $\mathbb{P}_{u, T}$. 


\section{Random walks on non-amenable graphs}

To prepare for the proof of Theorem 1.5 (2), this section recalls facts about random walks on non-amenable graphs.

Definition 5 (Simple random walk). For $x \in V$, let $P_{x}$ denote the law of the simple random walk started at $x$. Also let $\mathbb{E}_{x}$ denote expectation with respect to $\mathbb{P}_{x}$.

Definition 6 (Spectral radius). Let $\mathbf{w} \sim P_{x}$ and

$$
\rho:=\limsup _{n \rightarrow \infty} P_{x}(\mathbf{w}(n)=x)^{1 / n}
$$

be the spectral radius of the simple random walk. Because $G$ is connected, $\rho$ does not depend on the choice of $x$.

Definition 7 (Non-amenability). The graph $G$ is non-amenable if $\rho<1$. For example, if $G$ is a Cayley graph of a finitely generated group $\Gamma$ then $G$ is non-amenable if and only if $\Gamma$ is non-amenable [Woe00, Theorem 10.6].

The next lemma will be used in the sequel to obtain lower bounds on the growth of the level- $n$ random cluster $\mathcal{V}\left(\boldsymbol{\omega}_{n}^{x}\right)$.

Lemma 5.1. BNP11, Lemma 2.1] For any $K \Subset V$,

$$
\sum_{x \in K} P_{x}\left[\tilde{\mathrm{H}}_{K}=\infty\right] \geq(1-\rho) \# K
$$

Corollary 5.2. For any $K \Subset V$,

$$
\sum_{x \in K} P_{x}\left[\tilde{\mathrm{H}}_{K}=\infty\right]^{2} \geq(1-\rho)^{2} \# K
$$

Proof. By Cauchy-Schwartz and Lemma 5.1,

$$
\left(\sum_{x \in K} P_{x}\left[\tilde{\mathrm{H}}_{K}=\infty\right]^{2}\right)\left(\sum_{x \in K} 1\right) \geq\left(\sum_{x \in K} P_{x}\left[\tilde{\mathrm{H}}_{K}=\infty\right]\right)^{2} \geq(1-\rho)^{2}(\# K)^{2} .
$$

Dividing both sides by $\# K$ proves the corollary. 
Lemma 5.3. Suppose $G$ is non-amenable and connected. Then there exist constants $T_{0}, \delta_{0}, \sigma>$ 0 such that for every $x \in V$ if $T \geq T_{0}$ then

$$
P_{x}^{(T)}(\operatorname{len}(\mathbf{w}) \geq T, d(x, \mathbf{w}(T)) \geq \sigma T) \geq \delta_{0} .
$$

Proof. By [Woe00, Lemma $8.1(\mathrm{~b})]$, there is a constant $C>0$ such that

$$
P_{x}(\mathbf{w}(n)=y) \leq C \rho^{n}
$$

for all $x, y$. Summing over all $y$ in the ball of radius $\sigma n$ centered at $x$ yields

$$
\left.P_{x}(d(\mathbf{w}(n), x) \leq \sigma n)\right) \leq C D^{\sigma n} \rho^{n} .
$$

Choose $\sigma>0$ so that $D^{\sigma} \rho<1$ and $T_{0}$ so that $T \geq T_{0}$ implies $C D^{\sigma T} \rho^{T} \leq 1 / 2$. If $T \geq T_{0}$ then

$$
\begin{aligned}
P_{x}^{(T)}(\operatorname{len}(\mathbf{w}) \geq T, d(x, \mathbf{w}(T)) \geq \sigma T) & =P_{x}^{(T)}(d(x, \mathbf{w}(T)) \geq \sigma T \mid \operatorname{len}(\mathbf{w}) \geq T) P_{x}^{(T)}(\operatorname{len}(\mathbf{w}) \geq T) \\
& =P_{x}(d(x, \mathbf{w}(T)) \geq \sigma T) P_{x}^{(T)}(\operatorname{len}(\mathbf{w}) \geq T) \\
& \geq(1 / 2) P_{x}^{(T)}(\operatorname{len}(\mathbf{w}) \geq T)
\end{aligned}
$$

Note

$$
P_{x}^{(T)}(\operatorname{len}(\mathbf{w}) \geq T)=\frac{1}{T+1} \sum_{n \geq T}\left(\frac{T}{T+1}\right)^{n}=\left(\frac{T}{T+1}\right)^{T}=\left(1-\frac{1}{T+1}\right)^{T} \rightarrow e^{-1}
$$

as $T \rightarrow \infty$. So by choosing $T_{0}$ larger if necessary, we may assume $T \geq T_{0}$ implies $P_{x}^{(T)}(\operatorname{len}(\mathbf{w}) \geq T) \geq 1 / 3$. Set $\delta_{0}=1 / 6$ to finish the lemma.

\section{A truncated growth process}

The purpose of this section is to study a certain growth process related to $\left\{\mathcal{V}\left(\boldsymbol{\omega}_{n}^{x}\right)\right\}_{n}$. It is the main construction in our proof of Theorem 1.5 part (2) which is completed in the next section.

For simplicity, we will assume thoughout that $T$ is a natural number. We also make the following assumptions:

1. $G=(V, E)$ is a connected graph. 
2. $\operatorname{deg}_{x} \leq D$ for all $x \in V$ for some constant $D>0$.

3. $G$ is non-amenable. Let $\rho<1$ be its spectral radius and $\delta_{0}, \sigma, T_{0}$ be the constants in Lemma 5.3 .

4. Let $K \Subset L \Subset V$ be finite sets.

5. As in Corollary 4.2, for each $x \in L \backslash K$ let $\mathbf{N}_{x}$ be a Poisson random variable with mean $u \operatorname{deg}_{x}$ and $\left(\mathbf{a}_{x, i}, \mathbf{b}_{x, i}\right) \sim P_{x}^{(T)} \times P_{x}^{(T)}$ for $1 \leq i \leq \mathbf{N}_{x}$.

6. Let $\mathbf{R}^{\prime}$ be the set of all $x \in L \backslash K$ such that $\mathbf{N}_{x} \geq 1, \tilde{\mathrm{H}}_{L}\left(\mathbf{a}_{x, 1}\right)=\infty, H_{K}\left(\mathbf{b}_{x, 1}\right)=\infty$, $\operatorname{len}\left(\mathbf{a}_{x, 1}\right) \geq T$ and $d_{G}\left(\mathbf{a}_{x, 1}(T), x\right) \geq \sigma T$.

7. Let $\mathbf{a}_{x, i}^{\prime}$ be the walk defined by $\operatorname{len}\left(\mathbf{a}_{x, i}^{\prime}\right)=\min \left(T, \operatorname{len}\left(\mathbf{a}_{x, i}\right)\right)$ and

$$
\mathbf{a}_{x, i}^{\prime}(k)=\mathbf{a}_{x, i}(k) \quad 0 \leq k \leq \min \left(T, \operatorname{len}\left(\mathbf{a}_{x, i}\right)\right)
$$

8. Let $\boldsymbol{\omega}^{\prime}:=\sum_{x \in \mathbf{R}^{\prime}} \delta_{\mathbf{a}_{x, 1}^{\prime}}$.

The goal of this section is to prove:

Proposition 6.1. There exist constants $\delta_{1}, \delta_{2}>0$ depending only on $G$ such that if $T>T_{0}$, $\# K \leq(1-\rho)^{2} \# L / 2$ and $0<u<\delta_{1}$ then

$$
\mathbb{P}_{u, T}\left[\# \mathcal{V}\left(\boldsymbol{\omega}^{\prime}\right) \geq \delta_{2} u T \# L / 2\right] \geq 1-\frac{4 D^{3 T}}{u^{2} T^{2} \# L}
$$

To emphasize, the constants $\delta_{1}, \delta_{2}$ do not depend on $K, L, u$ or $T$.

Proposition 6.1 follows from Chebyshev's inequality once we have obtained appropriate bounds on the mean and variance of $\# \mathcal{V}\left(\boldsymbol{\omega}^{\prime}\right)$ which is the purpose of the next lemmas.

Lemma 6.2. There exists a universal constant $\delta_{1}^{\prime}>0$ such that if $0<u<\delta_{1}^{\prime}$ then

$$
(u / 2) \delta_{0}\left[(1-\rho)^{2} \# L-\# K\right] \leq \mathbb{E}\left[\# \mathbf{R}^{\prime}\right]
$$

Proof. By definition of $\mathbf{R}^{\prime}$,

$$
\mathbb{E}\left[\# \mathbf{R}^{\prime}\right]=\sum_{x \in L \backslash K} \mathbb{P}\left(x \in \mathbf{R}^{\prime}\right) .
$$


If $\mathbf{w} \sim \mathbb{P}_{x}^{(T)}$ then

$$
\begin{aligned}
& \mathbb{P}\left(x \in \mathbf{R}^{\prime}\right) \\
& =\mathbb{P}\left(\mathbf{N}_{x} \geq 1\right) P_{x}^{(T)}\left(\tilde{\mathrm{H}}_{L}(\mathbf{w})=\infty, \operatorname{len}(\mathbf{w}) \geq T, d_{G}(\mathbf{w}(T), x) \geq \sigma T\right) P_{x}^{(T)}\left(H_{K}(\mathbf{w})=\infty\right) .
\end{aligned}
$$

We assume $\delta_{1}^{\prime}>0$ is chosen so that if $u<\delta_{1}^{\prime}$ then $u / 2 \leq 1-\exp (-u)$. Assuming $u<\delta_{1}^{\prime}$ implies

$$
u / 2 \leq 1-\exp \left(-u \operatorname{deg}_{x}\right)=\mathbb{P}\left(\mathbf{N}_{x} \geq 1\right) .
$$

The next inequalities estimate the middle term in $\mathbb{P}\left(x \in \mathbf{R}^{\prime}\right)$ :

$$
\begin{aligned}
& P_{x}^{(T)}\left(\tilde{\mathrm{H}}_{L}\left(\mathbf{a}_{x, j}\right)=\infty, \operatorname{len}\left(\mathbf{a}_{x, j}\right) \geq T, d_{G}\left(\mathbf{a}_{x, j}^{\prime}(T), x\right) \geq \sigma T\right) \\
\geq & P_{x}^{(T)}\left(\tilde{\mathrm{H}}_{L}=\infty\right) P_{x}^{(T)}\left(\operatorname{len}\left(\mathbf{a}_{x, j}\right) \geq T, d_{G}\left(\mathbf{a}_{x, j}^{\prime}(T), x\right) \geq \sigma T\right) \\
\geq & P_{x}\left(\tilde{\mathrm{H}}_{L}=\infty\right) \delta_{0} .
\end{aligned}
$$

Finally, we estimate the last term in $\mathbb{P}\left(x \in \mathbf{R}^{\prime}\right)$

$$
P_{x}^{(T)}\left(H_{K}\left(\mathbf{b}_{x, j}\right)=\infty\right)=P_{x}^{(T)}\left(H_{K}=\infty\right) \geq P_{x}^{(T)}\left(\tilde{\mathrm{H}}_{L}=\infty\right) \geq P_{x}\left(\tilde{\mathrm{H}}_{L}=\infty\right) .
$$

Combine these estimates to obtain

$$
\begin{aligned}
\mathbb{E}\left[\# \mathbf{R}^{\prime}\right] & =\mathbb{E}\left[\sum_{x \in L \backslash K} \# \mathbf{R}_{x}^{\prime}\right] \geq(u / 2) \delta_{0} \sum_{x \in L \backslash K} P_{x}\left(\tilde{\mathrm{H}}_{L}=\infty\right)^{2} \\
& \geq(u / 2) \delta_{0}\left(\sum_{x \in L} P_{x}\left(\tilde{\mathrm{H}}_{L}=\infty\right)^{2}-\# K\right) \geq u \delta_{0}\left[(1-\rho)^{2} \# L-\# K\right]
\end{aligned}
$$

where the last inequality follows from Corollary 5.2.

Lemma 6.3. With notation as in Lemma 6.2. let

$$
\mathbf{I}=\left\{(x, y, t, s): x, y \in \mathbf{R}^{\prime}, x \neq y, t, s \in[0, T] \cap \mathbb{N}, \mathbf{a}_{x, 1}^{\prime}(t)=\mathbf{a}_{y, 1}^{\prime}(s)\right\}
$$

There is a constant $C>0$ such that $\mathbb{E}[\# \mathbf{I}] \leq C u^{2} \# L$.

Proof. The Greens function is defined by: $G(x, y)=\mathbb{E}_{x}[\#\{n \geq 0: \mathbf{w}(n)=y\}]$. Because $G$ is non-amenable, $G$ defines a bounded linear operator on $\ell^{2}(V)$ [Woe00, Theorem 10.3]. Consider running independent random walks $\mathbf{w}_{x}^{(i)}(i \in\{1,2\}, x \in L)$. The expected number 
of quadruples $(x, y, s, t)$ with $\mathbf{w}_{x}^{(1)}(s)=\mathbf{w}_{y}^{(2)}(t)$ is $\left\langle G 1_{L}, G 1_{L}\right\rangle$. In particular, conditioned on $\mathbf{R}^{\prime}$

$$
\# \mathbf{I} \leq\left\langle G 1_{\mathbf{R}^{\prime}}, G 1_{\mathbf{R}^{\prime}}\right\rangle
$$

The probability that a given vertex $x \in L$ is in $\mathbf{R}^{\prime}$ is bounded by $1-e^{-u D}$. So if we take the expectation in $\mathbf{R}^{\prime}$ of the above inequality, we obtain

$$
\mathbb{E}[\# \mathbf{I}] \leq\left(1-e^{-u D}\right)^{2}\left\langle G 1_{L}, G 1_{L}\right\rangle \leq\left(1-e^{-u D}\right)^{2}\|G\|^{2}\left\|1_{L}\right\|_{2}^{2} \leq u^{2} D^{2}\|G\|^{2} \# L
$$

Lemma 6.4. There are constants $\delta_{1}, \delta_{2}>0$ depending only on $G$ such that if $T>T_{0}$, $\# K \leq(1-\rho)^{2} \# L / 2$ and $0<u<\delta_{1}$ then

$$
\mathbb{E}\left[\# \mathcal{V}\left(\boldsymbol{\omega}^{\prime}\right)\right] \geq \delta_{2} u T \# L
$$

To emphasize, the constants $\delta_{1}, \delta_{2}$ do not depend on either $K, L, u$ or $T$.

Proof. For each $r \in \mathbf{R}^{\prime}, d\left(\mathbf{a}_{r}^{\prime}(0), \mathbf{a}_{r}^{\prime}(T)\right) \geq \sigma T$. So the total number of vertices in the range of $\mathbf{a}_{r}^{\prime}$ is at least $\sigma T$. It follows that

$$
\mathbb{E}\left[\# \mathcal{V}\left(\boldsymbol{\omega}^{\prime}\right)\right] \geq \sigma T \mathbb{E}\left[\# \mathbf{R}^{\prime}\right]-\mathbb{E}[\# \mathbf{I}]
$$

where $\mathbf{I}$ is as in Lemma 6.3. Assume $u \leq \delta_{1}^{\prime}$. So Lemmas 6.2 and 6.3 imply

$$
\mathbb{E}\left[\# \mathcal{V}\left(\boldsymbol{\omega}^{\prime}\right)\right] \geq \sigma T u \delta_{0}\left[(1-\rho)^{2} \# L-\# K\right] / 2-C u^{2} \# L
$$

So if $\# K \leq(1-\rho)^{2} \# L / 2$ and $u<C^{-1} \sigma T_{0} \delta_{0}(1-\rho)^{2} / 4$ then

$$
\mathbb{E}\left[\# \mathcal{V}\left(\boldsymbol{\omega}^{\prime}\right)\right] \geq \sigma T u \delta_{0}(1-\rho)^{2} \# L / 4=: \delta_{2} u T \# L
$$

where $\delta_{2}:=\sigma \delta_{0}(1-\rho)^{2} / 4$. Choose $\delta_{1}=\min \left(\delta_{1}^{\prime}, C^{-1} \sigma T_{0} \delta_{0}(1-\rho)^{2} / 4\right)$ to finish the lemma.

\section{Lemma 6.5.}

$$
\operatorname{Var}\left[\# \mathcal{V}\left(\boldsymbol{\omega}^{\prime}\right)\right] \leq D^{3 T} \# L
$$

Proof. Since $\# \mathcal{V}\left(\boldsymbol{\omega}^{\prime}\right)=\sum_{x \in V \backslash K} 1_{x \in \mathcal{V}\left(\boldsymbol{\omega}^{\prime}\right)}$,

$$
\operatorname{Var}\left[\# \mathcal{V}\left(\boldsymbol{\omega}^{\prime}\right)\right]=\sum_{x, y \in V \backslash K} \operatorname{Cov}\left(1_{x \in \mathcal{V}\left(\boldsymbol{\omega}^{\prime}\right)}, 1_{y \in \mathcal{V}\left(\boldsymbol{\omega}^{\prime}\right)}\right)
$$


where $\operatorname{Cov}(\cdot, \cdot)$ denotes covariance. Every walk in the support of $\boldsymbol{\omega}^{\prime}$ has length $T$. So if $d(x, y)>2 T$ then $1_{x \in \mathcal{V}\left(\boldsymbol{\omega}^{\prime}\right)}, 1_{y \in \mathcal{V}\left(\boldsymbol{\omega}^{\prime}\right)}$ are independent and $\operatorname{Cov}\left(1_{x \in \mathcal{V}\left(\boldsymbol{\omega}^{\prime}\right)}, 1_{y \in \mathcal{V}\left(\boldsymbol{\omega}^{\prime}\right)}\right)=0$. Also $x \in \mathcal{V}\left(\boldsymbol{\omega}^{\prime}\right)$ implies $x \in B_{T}(L)$, the radius $T$-neighborhood of $L$. Thus

$$
\operatorname{Var}\left[\# \mathcal{V}\left(\boldsymbol{\omega}^{\prime}\right)\right]=\sum_{x \in B_{T}(L) \backslash K} \sum_{d(x, y) \leq 2 T} \operatorname{Cov}\left(1_{x \in \mathcal{V}\left(\boldsymbol{\omega}^{\prime}\right)}, 1_{y \in \mathcal{V}\left(\boldsymbol{\omega}^{\prime}\right)}\right) .
$$

Since $1_{x \in \mathcal{V}\left(\boldsymbol{\omega}^{\prime}\right)}$ is either 0 or 1 ,

$$
\left|\operatorname{Cov}\left(1_{x \in \mathcal{V}\left(\boldsymbol{\omega}^{\prime}\right)}, 1_{y \in \mathcal{V}\left(\boldsymbol{\omega}^{\prime}\right)}\right)\right| \leq 1
$$

Therefore,

$$
\operatorname{Var}\left[\# \mathcal{V}\left(\boldsymbol{\omega}^{\prime}\right)\right] \leq \# B_{T}(L) D^{2 T} \leq D^{3 T} \# L
$$

Proposition 6.1 follows from Lemmas 6.4, 6.5 and Chebshev's inequality.

\subsection{At least one infinite cluster}

The next result strengthens Theorem 1.5 (2).

Theorem 6.6. Let $G=(V, E)$ be a connected bounded degree graph with spectral radius $\rho<1$. Then for every $u>0$ there exists $T_{u}>0$ such that if $T>T_{u}$ then for $\mathbb{P}_{u, T^{-}}$a.e. $\omega$, the subgraph $\left(V, \mathcal{E}_{\omega}\right)$ has infinite components.

Proof. Let $D$ be the maximum degree of a vertex in $G$. Let $T_{0}, \sigma, \delta_{0}, \delta_{1}, \delta_{2}>0$ be the constants from Lemmas 5.3 and 6.4 .

Recall that $\mathbb{P}_{u, T}$ is the law of the Poisson point process on $\Omega^{[0, \infty)}$ with intensity measure $u \nu^{(T)}$. So if $u_{1}, u_{2}>0$ are any positive numbers and $\boldsymbol{\omega}_{1}, \boldsymbol{\omega}_{2}$ are independent random variables with laws $\mathbb{P}_{u_{1}, T}, \mathbb{P}_{u_{2}, T}$ respectively, then $\boldsymbol{\omega}_{1}+\boldsymbol{\omega}_{2}$ has law $\mathbb{P}_{u_{1}+u_{2}, T}$. Since $\left(V, \mathcal{E}_{\boldsymbol{\omega}_{1}}\right)$ is a subgraph of $\left(V, \mathcal{E}_{\left.\boldsymbol{\omega}_{1}+\boldsymbol{\omega}_{2}\right)}\right)$ it follows that if $\left(V, \mathcal{E}_{\boldsymbol{\omega}}\right)$ has an infinite cluster for $\mathbb{P}_{u_{1}, T^{-}}$.e. $\omega$ then $\left(V, \mathcal{E}_{\omega}\right)$

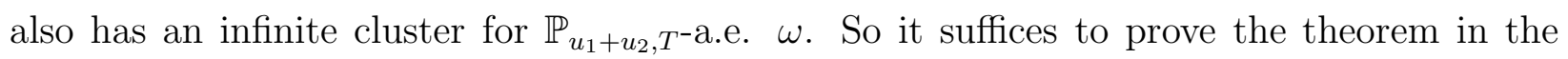
special case in which $0<u<\delta_{1}$. Assume this from now on.

Choose $T$ large enough so that $T>T_{0}$ and

$$
\left(1+\delta_{2} u T / 2\right) \geq \frac{2}{(1-\rho)^{2}} .
$$


Let $M \geq \frac{2}{(1-\rho)^{2}}$ be large enough so that

$$
\sum_{n=2}^{\infty} \frac{4 D^{3 T}}{u^{2} T^{2}\left(1+\delta_{2} u T / 2\right)^{n-2} M}<1 / 2 .
$$

Let $x \in V$ and recall the definition of $\omega_{n}^{x}$ from $\$ 4.1$. Also set $\omega_{0}^{x}$ equal to the zero measure. Define events $E_{n} \subset \Omega^{[0, \infty)}$ by

- $E_{1}$ is the subset of all $\omega \in \Omega^{[0, \infty)}$ such that $\# \mathcal{V}\left(\omega_{1}^{x}\right) \geq M$;

- for $n \geq 2, E_{n}$ is the subset of all $\omega \in \Omega^{[0, \infty)}$ such that $\# \mathcal{V}\left(\omega_{n}^{x}\right) \geq\left(1+\delta_{2} u T / 2\right) \# \mathcal{V}\left(\omega_{n-1}^{x}\right)$.

If $n \geq 2$ and $\omega \in \cap_{i<n} E_{i}$, then

$$
\# \mathcal{V}\left(\omega_{n-1}^{x}\right) \geq\left(1+\delta_{2} u T / 2\right)^{n-2} M
$$

and

$$
\# \mathcal{V}\left(\omega_{n-2}^{x}\right) \leq(1-\rho)^{2} \# \mathcal{V}\left(\omega_{n-1}^{x}\right) / 2
$$

Apply Proposition 6.1 with $K=\mathcal{V}\left(\omega_{n-2}^{x}\right), L=\mathcal{V}\left(\omega_{n-1}^{x}\right)$ to obtain

$$
\mathbb{P}_{u, T}\left(E_{n} \mid \cap_{i<n} E_{i}\right) \geq 1-\frac{4 D^{3 T}}{u^{2} T^{2} \# \mathcal{V}\left(\omega_{n-1}^{x}\right)} \geq 1-\frac{4 D^{3 T}}{u^{2} T^{2}\left(1+\delta_{2} u T / 2\right)^{n-2} M} .
$$

The reason this works is that if $\boldsymbol{\omega} \sim \mathbb{P}_{u, T}$ then $V_{n}(\boldsymbol{\omega}) \supset \mathcal{V}\left(\boldsymbol{\omega}^{\prime}\right)$ by Corollary 4.2 and Proposition 4.3. So

$$
\begin{aligned}
\mathbb{P}_{u, T}\left(\cap_{n \geq 1} E_{n}\right) & =\mathbb{P}_{u, T}\left(E_{1}\right) \prod_{n=2}^{\infty} \mathbb{P}_{u, T}\left(E_{n} \mid \cap_{i<n} E_{i}\right) \\
& \geq \mathbb{P}_{u, T}\left(E_{1}\right) \prod_{n=2}^{\infty} 1-\frac{4 D^{3 T}}{u^{2} T^{2}\left(1+\delta_{2} u T / 2\right)^{n-2} M} \\
& \geq \mathbb{P}_{u, T}\left(E_{1}\right)\left(1-\sum_{n=2}^{\infty} \frac{4 D^{3 T}}{u^{2} T^{2}\left(1+\delta_{2} u T / 2\right)^{n-2} M}\right) \\
& \geq \mathbb{P}_{u, T}\left(E_{1}\right) / 2>0 .
\end{aligned}
$$

For any $\omega \in \cap_{n \geq 1} E_{n}$ the cluster $\mathcal{V}\left(\omega_{\infty}^{x}\right)$ containing $x$ is infinite. So this proves the theorem. 


\section{$7 \quad$ Infinitely many infinite clusters}

The purpose of this section is to prove the following strengthening of Theorem [1.5 (3):

Theorem 7.1. Let $G=(V, E)$ be a vertex-transitive connected graph so that every vertex of $G$ has degree at most $D$. Let $u, T>0$ and suppose that $0<1+2 u D<\rho^{-1}$ where $\rho$ is the spectral radius. Also suppose that for $\mathbb{P}_{u, T}$-a.e. $\omega$, the subgraph $\left(V, \mathcal{E}_{\omega}\right)$ has infinite components. Then for $\mathbb{P}_{u, T}$-a.e. $\omega,\left(V, \mathcal{E}_{\omega}\right)$ has infinitely many infinite clusters.

First we show that the cluster at the identity is stochastically dominated by a specially designed multi-type branching random walk (mBRW). The trace of this mBRW coincides with a branching random walk (BRW) which is shown to be transient via [GM06]. The theorem then follows from a percolation-theory argument due to Benjamini-Schramm [BS11].

\subsection{A random colored forest}

Let $u, D>0$. We will construct a random colored forest $\mathcal{F}$ but first we describe its general structure. The vertex set, denoted $V^{\mathcal{F}}$, is a disjoint union $V^{\mathcal{F}}=\cup_{n \geq 1} V_{n}^{\mathcal{F}}$ and every edge is of the form $\left(v, v^{\prime}\right)$ with $v \in V_{n}^{\mathcal{F}}$ and $v^{\prime} \in V_{n+1}^{\mathcal{F}}$ for some $n$. In this case we call $v^{\prime}$ a child of $v$ and $v$ is the parent of $v^{\prime}$. We call $V_{n}^{\mathcal{F}}$ the $n$-th generation of the forest. Every vertex is assigned a color, either red, green or blue.

The forest is constructed as follow. The cardinality of the 1st generation is a Poisson random variable with mean $u D$. Every vertex in the 1 st generation is green.

Now suppose that the $n$-th generation has been construction. Then every green vertex has exactly two children, one red and one blue. Every red vertex has one red child, no blue children, and a Poisson $(u D)$-random number of green children. Similarly, every blue vertex has one blue child, no red children, and a Poisson $(u D)$-random number of green children. The random variables in the $n$-th stage of the construction are independent of each other and of all previously constructed random variables.

We denote the random colored forest by $\mathcal{F}=\left(V^{\mathcal{F}}, E^{\mathcal{F}}, \chi^{\mathcal{F}}\right)$ where $V^{\mathcal{F}}$ is the set of vertices, $E^{\mathcal{F}}$ is the set of edges and $\chi^{\mathcal{F}}: V^{\mathcal{F}} \rightarrow\{$ red,green, blue $\}$ is the coloring function. 


\subsection{The random map into $G$}

As usual, $G=(V, E)$ is a connected graph with all vertex degrees bounded by $D$. Fix $x \in V$. Let $\phi: V^{\mathcal{F}} \rightarrow V$ be the random map defined by:

- $\phi(v)=x$ for every $v$ in the first generation,

- If $\left(v, v^{\prime}\right) \in E^{\mathcal{F}}$ and $v^{\prime}$ is green then $\boldsymbol{\phi}(v)=\boldsymbol{\phi}\left(v^{\prime}\right)$

- If $\left(v, v^{\prime}\right) \in E^{\mathcal{F}}$ and $v^{\prime}$ is not green then $\phi\left(v^{\prime}\right)$ is a uniformly random neighbor of $\phi(v)$. Moreover, we require that the random variable $\phi\left(v^{\prime}\right)$ is conditionally independent of all previously constructed random variables (and all other random variables constructed on this stage) conditioned on $\phi(v)$.

The pair $(\mathcal{F}, \phi)$ is a multi-type Branching Random Walk (mBRW).

Lemma 7.2. Recall from 4.1 that $\mathbb{P}_{u, T}^{x}$ denotes the law of $\boldsymbol{\omega}_{\infty}^{x}$ where $\boldsymbol{\omega} \sim \mathbb{P}_{u, T}$. Let $G, x$ and $(\mathcal{F}, \phi)$ be the $m B R W$ constructed above. Then there exists a random variable $\tilde{\boldsymbol{\omega}} \sim \mathbb{P}_{u, T}^{x}$ taking values in $\Omega^{[0, \infty)}$ and coupled with $(\mathcal{F}, \phi)$ such that

- $\tilde{\boldsymbol{\omega}} \sim \mathbb{P}_{u, T}^{x}$,

- $\phi\left(E^{\mathcal{F}}\right) \supset \mathcal{E}(\tilde{\boldsymbol{\omega}})$ a.s.

Proof. Let $\mathcal{G} \subset V^{\mathcal{F}}$ be the subset of green vertices. Let $\left\{\boldsymbol{\tau}_{v}^{b}, \boldsymbol{\tau}_{v}^{r}\right\}_{v \in \mathcal{G}}$ be an i.i.d. family of random variables such that $\boldsymbol{\tau}_{v}^{b}+1 \sim \operatorname{Geom}(T+1)$ and $\boldsymbol{\tau}_{v}^{r}+1 \sim \operatorname{Geom}(T+1)$.

For each $v \in \mathcal{G}$, the red branch starting at $v_{0}$ is the unique path $R_{v}:=\left(v, R_{1}^{v}, \ldots, R_{k}^{v}\right)$ in the forest $\left(V^{\mathcal{F}}, E^{\mathcal{F}}\right)$ satisfying

- $\chi^{\mathcal{F}}\left(R_{i}^{v}\right)$ is red for all $1 \leq i \leq k$

- $\left(v, R_{1}^{v}\right)$ and $\left(R_{i}^{v}, R_{i+1}^{v}\right)$ are edges of $E^{\mathcal{F}}$ for all $i$

- $k=\boldsymbol{\tau}_{v}^{r}$

Blue branches are defined similarly. Let $\phi\left(R_{v}\right)=\left(\phi(v), \phi\left(R_{1}^{v}\right), \ldots, \phi\left(R_{k}^{v}\right)\right) \in \mathcal{W}[0, \infty)$ be the associated random walk. 
Let $\mathcal{G}_{1} \subset \mathcal{G}$ be the set of all green vertices $v \in V_{1}^{\mathcal{F}}$ such that $\tilde{\mathrm{H}}_{x}\left(\phi\left(R_{v}\right)\right)=\infty$. In other words, $\phi\left(R_{i}^{v}\right) \neq x$ for all $i>0$.

Let

$$
\tilde{\boldsymbol{\omega}}_{1}:=\sum_{v \in \mathcal{G}_{1}} \delta_{\operatorname{Con}\left(\phi\left(R_{v}\right), \phi\left(B_{v}\right)\right)}
$$

Also let $\boldsymbol{\omega} \sim \mathbb{P}_{u, T}$. By Proposition 4.3, the law of $\tilde{\boldsymbol{\omega}}_{1}$ is the same as the law of $\boldsymbol{\omega}_{1}^{x}$.

Suppose $\tilde{\boldsymbol{\omega}}_{n}$ and $\mathcal{G}_{n} \subset \mathcal{G} \cap V_{n}^{\mathcal{F}}$ have been defined so that $\tilde{\boldsymbol{\omega}}_{k}$ has the same law of $\boldsymbol{\omega}_{k}^{x}$ for all $1 \leq k \leq n$. Let $\mathcal{G}_{n+1}$ be the set of all vertices $v \in \mathcal{G} \cap V_{n+1}^{\mathcal{F}}$ such that

$$
\tilde{\mathrm{H}}_{\mathcal{V}\left(\tilde{\boldsymbol{\omega}}_{n}\right)}\left(\phi\left(R^{v}\right)\right)=\infty \text { and } H_{\mathcal{V}\left(\tilde{\boldsymbol{\omega}}_{n-1}\right)}\left(\phi\left(B^{v}\right)\right)=\infty
$$

In other words, $v \in \mathcal{G}_{n+1}$ if the image of the red branch does not return to $\mathcal{V}\left(\tilde{\boldsymbol{\omega}}_{n}\right)$ and the image of the blue branch does not traverse $\mathcal{V}\left(\tilde{\boldsymbol{\omega}}_{n-1}\right)$. Now let

$$
\tilde{\boldsymbol{\omega}}_{n+1}:=\tilde{\boldsymbol{\omega}}_{n}+\sum_{v \in \mathcal{G}_{n+1}} \delta_{\operatorname{Con}\left(\phi\left(R_{v}\right), \phi\left(B_{v}\right)\right)}
$$

By induction and Proposition 4.3, the law of $\tilde{\boldsymbol{\omega}}_{n+1}$ is the same as the law of $\boldsymbol{\omega}_{n+1}^{x}$. Let $\tilde{\boldsymbol{\omega}}=\sup _{n} \tilde{\boldsymbol{\omega}}_{n}$. By construction, $\tilde{\boldsymbol{\omega}} \sim \mathbb{P}_{u, T}^{x}$ and $\boldsymbol{\phi}\left(E^{\mathcal{F}}\right) \supset \mathcal{E}(\tilde{\boldsymbol{\omega}})$ a.s.

\subsection{Branching random walk}

The next step is to show that the image of the mBRW constructed above agrees with a certain branching random walk. Let $\theta$ be a probability distribution on $\mathbb{N}$. The Galton-Watson Tree with offspring distribution $\theta$ is the random tree $\mathcal{T}=\left(V^{\mathcal{T}}, E^{\mathcal{T}}\right)$ constructed as follows. As in the case of the random colored forest there is a partition $V^{\mathcal{T}}=\cup_{n \geq 0} V_{n}^{\mathcal{T}}$ of the vertices and every edge is of the form $\left(v, v^{\prime}\right)$ with $v \in V_{n}^{\mathcal{T}}, v^{\prime} \in V_{n+1}^{\mathcal{T}}$ for some $n$. In this case the index starts at 0 instead of starting at 1.

1. The 0 -th generation consists of a single individual.

2. Suppose the $n$-the generation has been constructed. Then every vertex of the $n$-th generation has a random number of children with law $\theta$. Moreover, these random variables are jointly independent and independent of all the random variables used in the construction of the $n$-th generation. 


\section{The Branching Random Walk (BRW) with offspring distribution $\theta$ started}

from $x \in V$ is a random pair $(\mathcal{T}, \boldsymbol{\psi})$ where

1. $\mathcal{T}$ is a Galton-Watson Tree with offspring distribution $\theta$,

2. $\boldsymbol{\psi}: \mathcal{T} \rightarrow G$ is a graph homomorphism,

3. $\boldsymbol{\psi}(v)=x$ where $v$ is the unique vertex in the 0 -th generation,

4. Suppose $\boldsymbol{\psi}(v)$ has been constructed for every vertex $v$ in the $n$-th generation of $\mathfrak{T}$. Then for every $v \in V_{n}^{\mathcal{T}}$ and $v^{\prime}$ with $\left(v, v^{\prime}\right) \in E^{\mathcal{T}}$, let $\boldsymbol{\psi}\left(v^{\prime}\right)$ be a uniformly random neighbor of $\boldsymbol{\psi}(v)$. Moreover, we require that the random variable $\boldsymbol{\psi}\left(v^{\prime}\right)$ is conditionally independent of all previously constructed random variables (and all other random variables constructed on this stage) conditioned on $\boldsymbol{\psi}(v)$.

The BRW is transient if almost surely $\boldsymbol{\psi}^{-1}(v)$ is finite for every $v \in V$. This does not depend on the choice of $x$ if $G$ is connected.

Theorem 3.2 of [GM06] implies

Lemma 7.3. If $\mathbf{X} \sim \theta, \mathbb{E}[\mathbf{X}] \leq \rho^{-1}$ and $\theta(\{0\})=0$ then the $B R W$ is transient.

Lemma 7.4. Let $G=(V, E)$ be a connected graph in which all vertex degrees are bounded by $D$. For any $u, T>0$ and $x \in V$ there exists a random tuple of variables $(\mathcal{F}, \boldsymbol{\phi}, \mathcal{T}, \boldsymbol{\psi})$ such that

- $(\mathcal{F}, \boldsymbol{\phi})$ is a $m B R W$ as in Lemma 7.2.

- $(\mathcal{T}, \boldsymbol{\psi})$ is a BRW started at $x$ with offspring distribution $\theta$ where, if $\mathbf{X} \sim \theta$ then $\frac{\mathbf{X}-1}{2} \sim$ $\operatorname{Poisson}(u D)$.

- the images $\boldsymbol{\psi}(\mathcal{T})$ and $\phi(\mathcal{F})$ agree.

Proof. Let $(\mathcal{F}, \phi)$ be a mBRW started at $x$ as in Lemma 7.2 . Let $\mathcal{G} \subset V^{\mathcal{F}}$ be the subset of green vertices. Let $\mathcal{T}^{\prime}$ be the tree obtained from $\mathcal{F}$ by adding one green vertex at the 0 -th generation and connecting it to every vertex in the 1st generation and then contracting every edge of the form $\left(v, v^{\prime}\right)$ such that $\chi^{\mathcal{F}}\left(v^{\prime}\right)=$ green. We view $\mathcal{T}^{\prime}$ as an uncolored tree. The law of $\mathcal{T}^{\prime}$ is almost the same as the Galton-Watson tree with offspring distribution. 
The difference is that the 1 st generation of $\mathcal{T}^{\prime}$ has cardinality $\sim 2$ Poisson $(u D)$ instead of $\sim 1+2$ Poisson $(u D)$. Otherwise, the laws are the same. Therefore, it is possible to couple $\mathcal{T}^{\prime}$ with $\mathcal{T}$ so that $\mathcal{T}^{\prime}$ is a subtree of $\mathcal{T}$.

Set $\boldsymbol{\psi}$ equal to $\boldsymbol{\phi}$ on $\mathcal{T}^{\prime}$. This is well-defined because every edge that gets contracted is mapped under $\phi$ to a single vertex. Extend $\boldsymbol{\psi}$ to all of $\mathcal{T}$ so that $(\mathcal{T}, \boldsymbol{\psi})$ is a BRW with offspring distribution $\theta$. The lemma now follows by construction.

Proof of Theorem 7.1. This argument is essentially the same as the proof of [BS11, Theorem $4]$.

Let $\theta$ be as in Lemma 7.4. Then the mean of $\theta$ is $1+2 u D<\rho^{-1}$. By Lemma 7.3, the BRW started at $x$ with offspring distribution $\theta$ is transient.

Let $\boldsymbol{\omega} \sim \mathbb{P}_{u, T}$. To obtain a contradiction suppose there are pairs of vertices $\left(x_{n}, y_{n}\right)$ and $\epsilon>0$ such that $d_{G}\left(x_{n}, y_{n}\right) \rightarrow \infty$ as $n \rightarrow \infty$ and yet the probability that $x_{n}$ and $y_{n}$ are in the same cluster of $\boldsymbol{\omega}$ is at least $\epsilon$ for all $n$. Since the BRW stochastically dominates the clusters, this means that with probability $\geq \epsilon$ BRW started from $x_{n}$ hits $y_{n}$ and with probability $\geq \epsilon$ BRW started from $y_{n}$ hits $x_{n}$. Consequently, with probability $\geq \epsilon^{2}$, the BRW started from $x_{n}$ will reach $x_{n}$ again after at least $d_{G}\left(x_{n}, y_{n}\right)$ time steps. Since $G$ is transitive, we may assume without loss of generality that all the $x_{n}$ 's are the same. But this contradicts transience of the BRW. This contradiction shows that for every $\epsilon>0$ there is a $k$ such that $d_{G}(x, y)>k$ implies that with probability $<\epsilon, x, y$ are in the same cluster of $\boldsymbol{\omega}$.

Let $r>0$ and consider $m$ balls of radius $r$ in $G$. If $r$ is large then the probability that each ball will intersect an infinite cluster of $\boldsymbol{\omega}$ nontrivially will be close to 1 . However, the probability that any infinite cluster of $\boldsymbol{\omega}$ intersects two balls tends to zero as the distance between the balls tends to infinity. It follows that, almost surely, there are at least $m$ infinite clusters of $\boldsymbol{\omega}$. Since $m$ is arbitrary, this proves the theorem.

Remark 3. The proof of Theorem 7.1 uses vertex-transitivity of $G$ only once, to assume that all of the $x_{n}$ 's are the same. This assumption can be removed as follows: let $x_{n}, y_{n}$ be as in the proof above. If $\left(G_{\infty}, x_{\infty}\right)$ is a sub-sequential Benjamini-Schramm limit of the rooted graphs $\left(G, x_{n}\right)$ then with probability $\geq \epsilon^{2}$, the BRW started at $x_{\infty}$ will reach $x_{\infty}$ again after $d_{G}\left(x_{n}, y_{n}\right)$ time steps for an infinite set of $n$ 's. In particular, the BRW is recurrent. However, the spectral radius of $G_{\infty}$ is bounded by the spectral radius of $G$ because $\rho$ is the 
supremum of $P_{x}(\mathbf{w}(n)=x)^{1 / n}$ as $n \rightarrow \infty$ and these functions are continuous with respect to Benjamini-Schramm convergence. This contradicts Lemma 7.3. The rest of the proof remains the same.

\section{Infinitely many ends and indistinguishability}

The purpose of this section is to prove the following slight strengthening of Theorem 1.5 (4):

Theorem 8.1. Let $u, T>0$ and $\boldsymbol{\omega} \sim \mathbb{P}_{u, T}$. Suppose $G$ has a transitive unimodular closed automorphism subgroup $\Gamma \leq \operatorname{Aut}(G)$. If $(V, \mathcal{E} \boldsymbol{\omega})$ has infinitely many infinite clusters a.s. then each infinite cluster has infinitely many ends a.s. Moreover $(V, \mathcal{E} \boldsymbol{\omega})$ has indistinguishable infinite clusters in the sense of [LS99].

Definition 8. Let $G=(V, E)$ be a graph. Given an edge $e \in E$ and a subset $A \subset E$, we let $\Pi_{e}(A):=A \cup\{e\}$. Given a collection $\mathcal{A} \subset 2^{E}$ of subsets, let $\Pi_{e}(\mathcal{A}):=\left\{\Pi_{e}(A): A \in \mathcal{A}\right\}$. Now suppose $\mathbf{S}$ is a random subset of $E$. We say that $\mathbf{S}$ is insertion-tolerant if for every edge $e \in E, \mathbb{P}(\mathbf{S} \in \mathcal{A})>0$ implies $\mathbb{P}\left(\mathbf{S} \in \Pi_{e}(\mathcal{A})\right)>0$.

Proposition 8.2. For any $u, T>0$, if $\boldsymbol{\omega} \sim \mathbb{P}_{u, T}$ then $\boldsymbol{\varepsilon}_{\boldsymbol{\omega}}$ is insertion tolerant.

Proof. This follows from the description of $\mathbb{P}_{u, T}$ as a Poisson point process.

Proof of Theorem 8.1. By [LS99, Theorem 3.3 and Proposition 3.10], insertion tolerance together with unimodular-transitivity of $G$ implies the conclusion.

Remark 4. Theorem 8.1 also holds for unimodular networks by [AL07, Corollary 6.11 and Theorem 6.15].

Theorem 1.5 follows from Theorems 3.1, 6.6, 7.1 and 8.1 .

\section{Proof of Theorem 1.1}

In this section, the final pieces are assembled to prove Theorem 1.1. We need to review standard facts from the theory of cost and treeability. 


\subsection{Cost and treeability}

Let $(X, \mu)$ be a standard probability space. A Borel equivalence relation $\mathcal{R} \subset X \times X$ is measure-preserving if for every Borel automorphism $\phi: X \rightarrow X$ such that $(x, \phi x) \in \mathcal{R}$ for every $x$ satisfies $\phi_{*} \mu=\mu$. The relation $\mathcal{R}$ is countable if its equivalence classes are countable. It is ergodic if for every measurable set $A \subset X$ that is a union of $\mathcal{R}$-classes, $\mu(A) \in\{0,1\}$. For $x \in X$, let $[x]_{\mathcal{R}} \subset X$ denote its $\mathcal{R}$-class.

By Feldman-Moore [FM77], $\mathcal{R}$ is a countable measure-preserving equivalence relation if and only if there is a countable group $\Gamma$ and a measure-preserving action $\Gamma \curvearrowright(X, \mu)$ such that $\mathcal{R}=\{(x, g x): x \in X, g \in \Gamma\}$ is the orbit-equivalence relation.

A graphing of $\mathcal{R}$ is a measurable set $\mathcal{G} \subset \mathcal{R}$ such that

- $(x, y) \in \mathcal{G} \Rightarrow(y, x) \in \mathcal{G}$

- for a.e. $x \in X$, the graph $\mathcal{G}_{x}$ with vertex set $[x]_{\mathcal{R}}$ and edges $\mathcal{G} \cap\left([x]_{\mathcal{R}} \times[x]_{\mathcal{R}}\right)$ is connected.

A graphing $\mathcal{G}$ is a treeing if for a.e. $x \in X$, the graph $\mathcal{G}_{x}$ is a tree. We say $\mathcal{R}$ is treeable if it admits a treeing.

The cost of a graphing $\mathcal{G}$ is

$$
\operatorname{cost}(\mathcal{G})=\frac{1}{2} \int \#\left\{y \in[x]_{\mathcal{R}}:(x, y) \in \mathcal{G}\right\} d \mu(x) .
$$

The cost of $\mathcal{R}$ is the infimum of $\operatorname{cost}(\mathcal{G})$ over all graphings $\mathcal{G}$ of $\mathcal{R}$.

The theory of cost was initiated by Levitt [Lev95] and developed into a powerful tool by Gaboriau [Gab00]. The main result we need is: if $\mathcal{G}$ is a treeing then $\mathcal{G}$ realizes the cost of $\mathcal{R}$ Gab00]. That is, $\operatorname{cost}(\mathcal{G})=\operatorname{cost}(\mathcal{R})$.

The next lemma will help us promote a treeing on part of $X$ to a treeing on all of $X$.

Lemma 9.1. Let $(X, \mu)$ be a standard probability space, $\mathcal{R} \subset X \times X$ a countable measurepreserving ergodic equivalence relation and $Y \subset X$ a subset with positive measure. Let $\mathcal{R}_{Y}=\mathcal{R} \cap Y \times Y$ be the restriction of $\mathcal{R}$ to $Y$. Suppose $\mathcal{S} \subset \mathcal{R}_{Y}$ is an ergodic, treeable subequivalence relation with cost $>1$ (with respect to the normalized measure $\mu(Y)^{-1} \mu(\cdot \cap Y)$ on $Y$ ). Then there exists an ergodic treeable subequivalence relation $\mathcal{S}^{\prime} \subset \mathcal{R}$ with cost $>1$.

Proof. Let $\mathcal{G}$ be a treeing of $\mathcal{S}$. Also let $\phi: X \backslash Y \rightarrow X$ be any measurable map such that 
1. for a.e. $x \in X \backslash Y, \phi(x) \in Y$

2. $(x, \phi(x)) \in \mathcal{R}$ for all $x$.

Such a map exists because $\mathcal{R}$ is ergodic. Define $\mathcal{G}^{\prime}$ to be the union of $\mathcal{G}$ with the edges $(x, \phi x)$ and $(\phi x, x)$ for $x \in X \backslash Y$. Also let $\mathcal{S}^{\prime}$ be the smallest subequivalence relation of $\mathcal{R}$ that contains $\mathcal{G}^{\prime}$. Then $\mathcal{G}^{\prime}$ is a treeing of $\mathcal{S}^{\prime}$. Moreover the cost of $\mathcal{S}^{\prime}$ is equal to $\mu(Y) \operatorname{cost}(\mathcal{S} \backslash Y)+\mu(X \backslash Y)$. This is because for every $x \in X \backslash Y$, we are adding an edge that adds one to the degree of $x$ and adds one to the degree of $\phi(x)$. Since $\operatorname{cost}(\mathcal{S}\lceil Y)>1$, this implies $\operatorname{cost}\left(\mathcal{S}^{\prime}\right)>1$.

In order to see that $\mathcal{S}^{\prime}$ is ergodic, let $A \subset X$ be a measurable union of $\mathcal{S}^{\prime}$ classes. Since $\mathcal{S}^{\prime} \cap$ $Y \times Y=\mathcal{S}, A \cap Y$ is a measurable union of $\mathcal{S}$-classes. Since $\mathcal{S}$ is ergodic, $\mu(A \cap Y) \in\{0, \mu(Y)\}$. For a.e. $x \in X$, the $\mathcal{S}^{\prime}$-class of $x$ intersects $Y$. So the fact that $\mu(A \cap Y) \in\{0, \mu(Y)\}$ implies $\mu(A) \in\{0,1\}$. Since $A$ is arbitrary, $\mathcal{S}^{\prime}$ is ergodic.

The next lemma will help us promote the weak vND on a factor action to the weak vND on the action.

Lemma 9.2. Suppose $\Gamma \curvearrowright(X, \mu)$ is an ergodic probability-measure-preserving action with an essentially free factor $\Gamma \curvearrowright(Y, \nu)$. If this factor is weakly vND then $\Gamma \curvearrowright(X, \mu)$ is also weakly $v N D$.

Proof. Because the factor is an essentially free action of $\Gamma$, the factor map $\Phi: X \rightarrow Y$ is class-bijective. This means that for a.e. $x \in X$, the restriction of $\Phi$ to the orbit $\Gamma x$ is a bijection onto the orbit $\Gamma \Phi(x)$.

Let $\mathbb{F}_{2} \curvearrowright(Y, \nu)$ be an essentially free, ergodic action of $\mathbb{F}_{2}$ whose orbits are contained in the orbit of the $\Gamma$-action. For $x \in X$ and $f \in \mathbb{F}_{2}$, define $f x \in \Gamma x$ to be the unique element such that $\Phi(f x)=f \Phi(x)$ where $\Phi: X \rightarrow Y$ is the factor map. This is well-defined for a.e. $x$ because $\Phi$ is class-bijective. Moreover it defines an essentially free action of $\mathbb{F}_{2}$ whose orbits are contained in the $\Gamma$-orbits.

\subsection{Strong solidity}

Strong solidity will be used to promote the weak vND to vND. 
Definition 9. Let $\Gamma \curvearrowright(X, \mu)$ be a probability-measure-preserving action and let $\mathcal{R}=\{(x, g x): x \in$ $X, g \in \Gamma\}$ be the orbit-equivalence relation. Then the action is said to be solidly ergodic if for any measurable subequivalence relation $\mathcal{S} \subset \mathcal{R}$ there exists a measurable partition of $X$ into countably many pieces $X=\sqcup_{i=0}^{\infty} X_{i}$ such that

1. each $X_{i}$ is union of $\mathcal{S}$-classes,

2. $\mathcal{S} \uparrow X_{0}$ is hyperfinite,

3. and $\mathcal{S}\left\lceil X_{i}\right.$ is strongly ergodic for all $i>0$.

This concept was introduced in [CI10] and given its name in [Gab10].

Proposition 9.3. Let $\Gamma \curvearrowright(X, \mu)$ be an essentially free, solidly ergodic action. If this action is weakly $v N D$ then it is $v N D$.

Proof. Without loss of generality, we may assume $\Gamma$ is non-amenable. Let $\mathcal{R}$ be the orbitequivalence relation of this action. By assumption there exists an essentially free action $\mathbb{F}_{2} \curvearrowright(X, \mu)$ such that if $\mathcal{S}=\left\{(x, f x): x \in X, f \in \mathbb{F}_{2}\right\}$ then $\mathcal{S} \subset \mathcal{R}$. Because the action is solidly ergodic, there exists a measurable partition of $X$ into countably many pieces $X=$ $\sqcup_{i=0}^{\infty} X_{i}$ such that $\mathcal{S} \uparrow X_{0}$ is hyperfinite and $\mathcal{S} \uparrow X_{i}$ is strongly ergodic for all $i>0$. Because $\mathbb{F}_{2}$ is non-amenable, $X_{0}$ must be have measure zero. Without loss of generality, $\mu\left(X_{1}\right)>0$. By Lemma 9.1 there exists an ergodic treeable subequivalence relation $\mathcal{S}^{\prime} \subset \mathcal{R}$ with cost $>1$. By [GL09, Proposition 14], $\mathcal{S}^{\prime}$ contains a subrelation that is the orbit-equivalence relation for an ergodic essentially free action of a rank 2 free group. This last result uses Hjorth's Lemma Hjo06.

\subsection{The final step}

We will need a recent result of Seward:

Theorem 9.4 ([Sew18]). Let $\Gamma$ be a countably infinite group and $(K, \kappa),(L, \lambda)$ probability spaces with the same Shannon entropies $H(\kappa)=H(\lambda)$. Then the corresponding Bernoulli shifts $\Gamma \curvearrowright(K, \kappa)^{\Gamma}$ and $\Gamma \curvearrowright(L, \lambda)^{\Gamma}$ are isomorphic. 
Corollary 9.5. Let $\Gamma$ be a countably infinite group and $(K, \kappa),(L, \lambda)$ probability spaces with $H(\kappa) \geq H(\lambda)$. Then $\Gamma \curvearrowright(K, \kappa)^{\Gamma}$ factors onto $\Gamma \curvearrowright(L, \lambda)^{\Gamma}$.

Proof. Let $(N, \nu)$ be another probability space with

$$
H(\kappa)=H(\lambda)+H(\nu)=H(\lambda \times \nu) .
$$

By Theorem 9.4, $\Gamma \curvearrowright(K, \kappa)^{\Gamma}$ is measurably conjugate to $\Gamma \curvearrowright(L \times N, \lambda \times \nu)^{\Gamma}$. The projection map $(L \times N)^{\Gamma} \rightarrow L^{\Gamma}$ gives the desired factor.

Proof of Theorem 1.1. Without loss of generality, we may assume $\Gamma$ is non-amenable. Because amenability is closed under direct unions, every non-amenable group $\Gamma$ contains a finitely generated non-amenable subgroup $\Gamma^{\prime}$. Any Bernoulli shift action $\Gamma \curvearrowright(K, \kappa)^{\Gamma}$ restricts to a Bernoulli action of $\Gamma^{\prime}$. So we may assume without loss of generality that $\Gamma$ is finitely generated.

By [CI10] every Bernoulli shift action of $\Gamma$ is solidly ergodic. By Proposition 9.3 and Lemma 9.2 it suffices to show that every Bernoulli action $\Gamma \curvearrowright(K, \kappa)^{\Gamma}$ admits an essentially free factor $\Gamma \curvearrowright(X, \mu)$ that is weakly vND. Because Bernoulli shifts are mixing, every nontrivial factor of a Bernoulli shift is essentially free. By Corollary 9.5, it suffices to prove that for every $\epsilon>0$ there exists a Bernoulli shift $\Gamma \curvearrowright(K, \kappa)^{\Gamma}$ with $H(\kappa)<\epsilon$ such that this Bernoulli shift admits a nontrivial factor that is weakly vND.

Let $G=(V, E)$ be a Cayley graph of $\Gamma$. By Theorem 1.5 there exist $u, T>0$ such that $\Gamma \curvearrowright\left(\Omega, \mathbb{P}_{u, T}\right)$ is measurably conjugate to a Bernoulli shift with base entropy $<\epsilon$ and for $\mathbb{P}_{u, T}$-a.e. $\omega,\left(V, \mathcal{E}_{\omega}\right)$ has infinitely many infinite clusters with infinitely many ends. Moreover, $\left(V, \mathcal{E}_{\boldsymbol{\omega}}\right)$ has indistiguishable infinite clusters (where $\boldsymbol{\omega} \sim \mathbb{P}_{u, T}$ ).

The rest of the proof is essentially the same as in GL09. Let $\Gamma$ act on $E$ by lefttranslation. This induces an action on $2^{E}$, the space of all subsets of $E$ with the pointwise convergence topology. The map $\omega \mapsto \mathcal{E}_{\omega}$ from $\Omega^{[0, \infty)}$ to $2^{E}$ is $\Gamma$-equivariant. So $\mathcal{E}_{*} \mathbb{P}_{u, T}$ is an invariant measure on $2^{E}$. Let $\mathcal{R}=\left\{(\omega, g \omega): \omega \in 2^{E}, g \in \Gamma\right\}$ be the orbit-equivalence relation.

Let $X_{\infty} \subset 2^{E}$ be the set of $\omega \subset E$ such that the identity element is contained in an infinite cluster of $\left(V, \mathcal{E}_{\omega}\right)$. The cluster relation, denoted $\mathcal{R}^{c l} \subset \mathcal{R}$, is the set of all pairs $(\omega, g \omega)$ such that the component of the graph $\left(V, \mathcal{E}_{\omega}\right)$ containing the identity element also 
contains $g^{-1}$. Because $\left(V, \mathcal{E}_{\boldsymbol{\omega}}\right)$ has indistinguishable infinite clusters, $\mathcal{R}^{c l} \uparrow X_{\infty}$ is ergodic with respect to the measure $\mathcal{E}_{*} \mathbb{P}_{u, T}$.

By Gab00, Cor. IV.24 (2)], because the cluster containing the origin has infinitely many ends with positive probability, the cost of $\mathcal{R}^{c l} \uparrow X_{\infty}$ is larger than 1 . By either [KM04, Lem.28.11; 28.12] or [Pic05, Corollary 40], $\mathcal{R}^{c l} \uparrow X_{\infty}$ contains an ergodic treeable subequivalence relation $\mathcal{F}$ with cost larger than 1 (this is obtained from an arbitrary graphing by carefully removing cycles). By Lemma 9.1, there is an ergodic treeable equivalence relation $\mathcal{S} \subset \mathcal{R}$ with cost $>1$. By [GL09, Proposition 14], $\mathcal{S}$ contains a subrelation that is the orbitequivalence relation for an ergodic essentially free action of a rank 2 free group. This last result uses Hjorth's Lemma Hjo06.

So the action $\mathbb{F}_{2} \curvearrowright\left(2^{E}, \mathcal{E}_{*} \mathbb{P}_{u, T}\right)$ is vND. Since this is a factor of the Bernoulli action $\Gamma \curvearrowright\left(\Omega, \mathbb{P}_{u, T}\right)$ which has base entropy $<\epsilon$, this completes the proof.

\section{Applications}

\subsection{Actions with positive Rokhlin entropy}

This section proves Theorem 1.2 .

Definition 10. Fix a standard probability space $(X, \mu)$. The Shannon entropy of a countable measurable partition $\mathcal{P}$ of $X$ is defined by

$$
H_{\mu}(\mathcal{P}):=-\sum_{P \in \mathcal{P}} \mu(P) \log \mu(P)
$$

A partition $\mathcal{P}$ is generating for an action $\Gamma \curvearrowright(X, \mu)$ if the smallest $\Gamma$-invariant sigma-algebra containing $\mathcal{P}$ contains all Borel subsets (up to measure zero). The Rokhlin entropy of an ergodic probability-measure-preserving action $\Gamma \curvearrowright(X, \mu)$ is defined to be the infimum of $H_{\mu}(\mathcal{P})$ over all generating partitions for the action.

Seward's generalization of Sinai's Factor Theorem [Sew15] is:

Theorem 10.1 (Seward's Factor Theorem). Let $\Gamma$ be a countably infinite group and let $\Gamma \curvearrowright(X, \mu)$ be an essentially free, probability-measure-preserving, ergodic action with positive Rokhlin entropy. Then $\Gamma \curvearrowright(X, \mu)$ factors onto a Bernoulli shift. 
Corollary 1.2 follows immediately from Theorem 1.1, Theorem 10.1 and Lemma 9.2 .

\subsection{Factors of Bernoulli shifts}

In this section, we provide details to explain Corollaries 1.3 and 1.4. We will need the following lemma proven in [Bow16, Lemma 6].

Lemma 10.2. Let $\Gamma \curvearrowright(X, \mu)$ be an essentially free factor of a Bernoulli shift and suppose that its orbit-equivalence relation contains a non-hyperfinite treeable subequivalence relation $\mathcal{S}$. Then for every pair of probability spaces $(K, \kappa),(L, \lambda)$ the direct product action

$$
\Gamma \curvearrowright\left(X \times K^{\Gamma}, \mu \times \kappa^{\Gamma}\right)
$$

factors onto the Bernoulli shift $\Gamma \curvearrowright(L, \lambda)^{\Gamma}$.

Proof of Corollary 1.3. Let $(K, \kappa),(L, \lambda)$ be nontrivial standard probability spaces. For $i=$ 1,2 , let $\left(K_{i}, \kappa_{i}\right)$ be nontrivial standard probability spaces such that

$$
H(\kappa)=H\left(\kappa_{1}\right)+H\left(\kappa_{2}\right) .
$$

By Theorem 9.4, $\Gamma \curvearrowright(K, \kappa)^{\Gamma}$ is measurably conjugate to $\Gamma \curvearrowright\left(K_{1} \times K_{2}, \kappa_{1} \times \kappa_{2}\right)^{\Gamma}$ which in turn is isomorphic to the direct product action $\Gamma \curvearrowright\left(K_{1}, \kappa_{1}\right)^{\Gamma} \times\left(K_{2}, \kappa_{2}\right)^{\Gamma}$. By Theorem 1.1 and Lemma 10.2, this implies that $\Gamma \curvearrowright(K, \kappa)^{\Gamma}$ factors onto $\Gamma \curvearrowright(L, \lambda)^{\Gamma}$.

Corollary 10.3. Let $\Gamma$ be a non-amenable sofic group. Then the set of all nontrivial factors of Bernoulli shifts over $\Gamma$ forms a single weak isomorphism class.

Proof. It is immediate from the definition of sofic entropy that Rokhlin entropy upper bounds sofic entropy [Bow17]. By Ker14 any nontrivial factor $\Gamma \curvearrowright(X, \mu)$ of a Bernoulli shift has positive sofic entropy and therefore positive Rokhlin entropy. Seward's Factor Theorem 10.1 implies that $\Gamma \curvearrowright(X, \mu)$ factors onto a Bernoulli shift. By Theorem 1.3, $\Gamma \curvearrowright(X, \mu)$ factors onto all Bernoulli shifts and therefore $\Gamma \curvearrowright(X, \mu)$ factors onto all factors of all Bernoulli shifts.

Remark 5. Popa and Sasyk showed that if $\Gamma$ has property $(\mathrm{T})$ then there exist factors of Bernoulli shifts over $\Gamma$ that are not isomorphic to Bernoulli shifts [PS07].

Corollary 1.4 is an immediate corollary of Theorem 10.1 and Corollary 1.3. 


\section{A Convergence to RI}

This section shows how FRI converges to the random interlacement process (RI) in distribution as $T \rightarrow \infty$. We will use notation as in [Hut18, §3.2] to define the RI. The proof of Theorem A.2 below is similar in spirit to [Hut18, Proposition 3.3].

For $K \subset V$ and $w \in \mathcal{W}[m, n]$, let

$$
H_{K}^{+}(w)=\sup \{m \leq i \leq n: w(i) \in K\}
$$

For $w \in \mathcal{W}_{K}$, let $w_{K}$ be the restriction of $w$ to the interval $\left[H_{K}(w), H_{K}^{+}(w)\right]$. We equip $\mathcal{W}$ with the topology generated by open sets of the form

$$
\left\{w \in \mathcal{W}_{K}: w_{K}=w_{K}^{\prime}\right\}
$$

where $K \subset V$ is finite and $w^{\prime} \in \mathcal{W}_{K}$. This is a complete Polish topology although it is non-compact if $V$ is infinite since there are infinitely many restrictions $w_{K}^{\prime}$.

The time shift $\theta_{k}: \mathcal{W} \rightarrow \mathcal{W}$ is defined by $\theta_{k}: \mathcal{W}[m, n] \rightarrow \mathcal{W}[m-k, n-k]$,

$$
\theta_{k}(w)(i)=w(i+k)
$$

The space $\mathcal{W}^{*}$ is defined to be the quotient $\mathcal{W}^{*}=\mathcal{W} / \sim$ where $w_{1} \sim w_{2}$ if and only if $w_{1}=\theta_{k}\left(w_{2}\right)$ for some $k$. Let $\pi: \mathcal{W} \rightarrow \mathcal{W}^{*}$ denote the quotient map and equip $\mathcal{W}$ with the quotient topology.

For $w \in \mathcal{W}$, let $w^{\leftarrow} \in \mathcal{W}$ be the time reversed walk defined by $w^{\leftarrow}(i)=w(-i)$. For $\mathscr{A} \subset \mathcal{W}$, let $\mathscr{A}^{\leftarrow}=\left\{w^{\leftarrow}: w \in \mathscr{A}\right\}$. If $w \in \mathcal{W}[m, n]$ and $m \leq m^{\prime} \leq n^{\prime} \leq n$ then let $w \uparrow\left[m^{\prime}, n^{\prime}\right] \in \mathcal{W}\left[m^{\prime}, n^{\prime}\right]$ be the restriction.

For a finite set $K \subset V$, let $Q_{K}$ be the measure on $\mathcal{W}$ defined by

$$
Q_{K}(\{w \in \mathcal{W}: w(0) \notin K\})=0
$$

and for each $x \in K$ and Borel subsets $\mathscr{A}, \mathscr{B} \subset \mathcal{W}$,

$$
\begin{aligned}
& Q_{K}(\{w \in \mathcal{W}: w \uparrow(-\infty, 0] \in \mathscr{A}, w(0)=x \text { and } w \uparrow[0, \infty) \in \mathscr{B}\}) \\
= & \operatorname{deg}_{x} P_{x}\left(\mathscr{A}^{\leftarrow} \cap \tilde{\mathrm{H}}_{K}=\infty\right) P_{x}(\mathscr{B}) .
\end{aligned}
$$


Theorem A.1 (Sznitman [Szn10] and Teixeira [Tei09]). Let $G$ be a transient graph. There exists a unique $\sigma$-finite measure $Q^{*}$ on $\mathcal{W}^{*}$ such that for every Borel set $\mathscr{A} \subset \mathcal{W}^{*}$ and finite $K \subset V$,

$$
Q^{*}\left(\mathscr{A} \cap \pi\left(\mathcal{W}_{K}\right)\right)=Q_{K}\left(\pi^{-1}(\mathscr{A})\right) .
$$

Let $\Omega^{*}$ be the set of locally finite counting measures on $\mathcal{W}^{*}$ with the weak* topology. This means that measures $\omega_{i} \in \Omega^{*}$ converge to $\omega_{\infty} \in \Omega^{*}$ iff for every finite $K \subset V$ and $w^{\prime} \in \mathcal{W}_{K}$,

$$
\omega_{i}\left(\pi\left(\left\{w \in \mathcal{W}_{K}: w_{K}=w_{K}^{\prime}\right\}\right)\right) \rightarrow \omega_{\infty}\left(\pi\left(\left\{w \in \mathcal{W}_{K}: w_{K}=w_{K}^{\prime}\right\}\right)\right) .
$$

A random interlacement with intensity $u$ is a Poisson point process with intensity measure $u Q^{*}$. Let $\mathbb{P}_{u}^{*}$ be its law.

Theorem A.2. For any $u>0$, the measures $\mathbb{P}_{u, T} \circ \pi^{-1}$ converge to $\mathbb{P}_{u}^{*}$ in the weak ${ }^{*}$ topology as $T \rightarrow \infty$.

Proof. By Proposition 4.1 and Theorem A.1, it suffices to show for every finite $K \subset V$, $\mathrm{Con}_{*} Q_{K, \emptyset}^{(T)}$ converges to $Q_{K}$ as $T \rightarrow \infty$. This follows from the fact that $\mathbb{P}_{x}^{(T)}$ converges to $\mathbb{P}_{x}$ as $T \rightarrow \infty$ (for any $x \in V$ ).

\section{References}

[AL07] David Aldous and Russell Lyons. Processes on unimodular random networks. Electron. J. Probab., 12:no. 54, 1454-1508, 2007.

[Bal05] Karen Ball. Factors of independent and identically distributed processes with nonamenable group actions. Ergodic Theory Dynam. Systems, 25(3):711-730, 2005.

[BHI18] Lewis Bowen, Daniel Hoff, and Adrian Ioana. von Neumann's problem and extensions of non-amenable equivalence relations. Groups Geom. Dyn., 12(2):399-448, 2018.

[BNP11] Itai Benjamini, Asaf Nachmias, and Yuval Peres. Is the critical percolation probability local? Probab. Theory Related Fields, 149(1-2):261-269, 2011. 
[Bow10] Lewis Bowen. Measure conjugacy invariants for actions of countable sofic groups. J. Amer. Math. Soc., 23(1):217-245, 2010.

[Bow11] Lewis Bowen. Weak isomorphisms between Bernoulli shifts. Israel J. Math., 183:93$102,2011$.

[Bow12] Lewis Bowen. Every countably infinite group is almost Ornstein. In Dynamical systems and group actions, volume 567 of Contemp. Math., pages 67-78. Amer. Math. Soc., Providence, RI, 2012.

[Bow16] Lewis Bowen. Zero entropy is generic. to appear in a special edition of Entropy, 2016.

[Bow17] Lewis Bowen. Examples in the entropy theory of countable group actions. submitted, 2017.

[Bow18] Lewis Bowen. Equivalence relations that act on bundles of hyperbolic spaces. Ergodic Theory Dynam. Systems, 38(7):2447-2492, 2018.

[BS11] Itai Benjamini and Oded Schramm. Percolation beyond $\mathbb{Z}^{d}$, many questions and a few answers [mr1423907]. In Selected works of Oded Schramm. Volume 1, 2, Sel. Works Probab. Stat., pages 679-690. Springer, New York, 2011.

[CI10] Ionut Chifan and Adrian Ioana. Ergodic subequivalence relations induced by a Bernoulli action. Geom. Funct. Anal., 20(1):53-67, 2010.

[CT06] Thomas M. Cover and Joy A. Thomas. Elements of information theory. WileyInterscience [John Wiley \& Sons], Hoboken, NJ, second edition, 2006.

[DRS14] Alexander Drewitz, Balázs Ráth, and Artëm Sapozhnikov. An introduction to random interlacements. SpringerBriefs in Mathematics. Springer, Cham, 2014.

[FM77] Jacob Feldman and Calvin C. Moore. Ergodic equivalence relations, cohomology, and von Neumann algebras. I. Trans. Amer. Math. Soc., 234(2):289-324, 1977.

[Gab00] Damien Gaboriau. Coût des relations d'équivalence et des groupes. Invent. Math., 139(1):41-98, 2000. 
[Gab02] Damien Gaboriau. Arbres, groupes, quotients. Habilitation Thesis, École Normale Supérieure de Lyon, 2002.

[Gab10] Damien Gaboriau. Orbit equivalence and measured group theory. In Proceedings of the International Congress of Mathematicians. Volume III, pages 1501-1527. Hindustan Book Agency, New Delhi, 2010.

[GL09] Damien Gaboriau and Russell Lyons. A measurable-group-theoretic solution to von Neumann's problem. Invent. Math., 177(3):533-540, 2009.

[GM06] N. Gantert and S. Müller. The critical branching Markov chain is transient. Markov Process. Related Fields, 12(4):805-814, 2006.

[Hjo06] Greg Hjorth. A lemma for cost attained. Ann. Pure Appl. Logic, 143(1-3):87-102, 2006.

[Hou12] Cyril Houdayer. Invariant percolation and measured theory of nonamenable groups [after Gaboriau-Lyons, Ioana, Epstein]. Astérisque, (348):Exp. No. 1039, ix, 339374, 2012. Séminaire Bourbaki: Vol. 2010/2011. Exposés 1027-1042.

[Hut18] Tom Hutchcroft. Interlacements and the wired uniform spanning forest. Ann. Probab., 46(2):1170-1200, 2018.

[Ker14] David Kerr. Bernoulli actions of sofic groups have completely positive entropy. Israel J. Math., 202(1):461-474, 2014.

[KL11] David Kerr and Hanfeng Li. Bernoulli actions and infinite entropy. Groups Geom. Dyn., 5(3):663-672, 2011.

[KM04] Alexander S. Kechris and Benjamin D. Miller. Topics in orbit equivalence, volume 1852 of Lecture Notes in Mathematics. Springer-Verlag, Berlin, 2004.

[Kun13] Gábor Kun. Expanders have a spanning lipschitz subgraph with large girth. arXiv preprint arXiv:1303.4982, 2013.

[Lev95] Gilbert Levitt. On the cost of generating an equivalence relation. Ergodic Theory Dynam. Systems, 15(6):1173-1181, 1995. 
[LS99] Russell Lyons and Oded Schramm. Indistinguishability of percolation clusters. Ann. Probab., 27(4):1809-1836, 1999.

[Ol'91] A. Yu. Ol'shanskil. Geometry of defining relations in groups, volume 70 of Mathematics and its Applications (Soviet Series). Kluwer Academic Publishers Group, Dordrecht, 1991. Translated from the 1989 Russian original by Yu. A. Bakhturin.

[Orn70] Donald Ornstein. Bernoulli shifts with the same entropy are isomorphic. Advances in Math., 4:337-352 (1970), 1970.

[OW80] Donald S. Ornstein and Benjamin Weiss. Ergodic theory of amenable group actions. I. The Rohlin lemma. Bull. Amer. Math. Soc. (N.S.), 2(1):161-164, 1980.

[OW87] Donald S. Ornstein and Benjamin Weiss. Entropy and isomorphism theorems for actions of amenable groups. J. Analyse Math., 48:1-141, 1987.

[Pic05] Mikaël Pichot. Quasi-périodicité et théorie de la mesure. Ph.D. Thesis, École Normale Supérieure de Lyon, 2005.

[PS07] Sorin Popa and Roman Sasyk. On the cohomology of Bernoulli actions. Ergodic Theory Dynam. Systems, 27(1):241-251, 2007.

[Sew15] Brandon Seward. Positive entropy actions of countable groups factor onto Bernoulli shifts. to appear in the Journal of the AMS, 2015.

[Sew18] Brandon Seward. Bernoulli shifts with bases of equal entropy are isomorphic. arXiv:1805.08279, 2018.

[Ste75] A. M. Stepin. Bernoulli shifts on groups. Dokl. Akad. Nauk SSSR, 223(2):300-302, 1975.

[Szn10] Alain-Sol Sznitman. Vacant set of random interlacements and percolation. Ann. of Math. (2), 171(3):2039-2087, 2010.

[Tei09] A. Teixeira. Interlacement percolation on transient weighted graphs. Electron. J. Probab., 14:no. 54, 1604-1628, 2009. 
[Tit72] J. Tits. Free subgroups in linear groups. J. Algebra, 20:250-270, 1972.

[TT13] Augusto Teixeira and Johan Tykesson. Random interlacements and amenability. Ann. Appl. Probab., 23(3):923-956, 2013.

[Woe00] W. Woess. Random walks on infinite graphs and groups, volume 138. Cambridge university press, 2000. 